\title{
Balancing torques in membrane-mediated interactions: Exact results and numerical illustrations
}

\author{
Martin Michael Müller and Markus Deserno \\ Max-Planck-Institut für Polymerforschung, Ackermannweg 10, 55128 Mainz, Germany \\ Jemal Guven \\ Instituto de Ciencias Nucleares, Universidad Nacional Autónoma de México, Apdo. Postal 70-543, 04510 México D.F., Mexico
}

(Received 9 February 2007; published 26 July 2007)

\begin{abstract}
Torques on interfaces can be described by a divergence-free tensor which is fully encoded in the geometry. This tensor consists of two terms, one originating in the couple of the stress, the other capturing an intrinsic contribution due to curvature. In analogy to the description of forces in terms of a stress tensor, the torque on a particle can be expressed as a line integral along a contour surrounding the particle. Interactions between particles mediated by a fluid membrane are studied within this framework. In particular, torque balance places a strong constraint on the shape of the membrane. Symmetric two-particle configurations admit simple analytical expressions which are valid in the fully nonlinear regime; in particular, the problem may be solved exactly in the case of two membrane-bound parallel cylinders. This apparently simple system provides some flavor of the remarkably subtle nonlinear behavior associated with membrane-mediated interactions.
\end{abstract}

DOI: 10.1103/PhysRevE.76.011921

PACS number(s): 87.16.Dg, 68.03.Cd, 02.40.Hw

\section{INTRODUCTION}

The crucial role of fluid membranes as a component of living cells was recognized early on by biologists [1]. In recent years, the mechanics of membranes has become a fertile area of research among physicists as well [2]. Many cellular tasks such as exocytosis or endocytosis [3], the formation of vesicles [4-6], or the interaction with the cytoskeleton [7] rely heavily on mechanical membrane properties and can be studied using the mathematical toolbox of theoretical physics. Various key questions are of an essentially geometrical nature: what is the shape adopted by a membrane subjected to some specified boundary conditions [8]? How are forces and torques transmitted through the membrane [9-12]? Which interactions does this imply [13-24]?

To answer questions such as these, one approach is to apply an effective formulation in which the membrane is modeled as a two-dimensional surface in space. This approach is valid provided the length scales of interest are much longer than the membrane thickness which typically is a few nanometers. The energy of the membrane is then completely determined by the geometry of the surface. In distinction to a simple fluid-fluid interface where the energy is dominated by surface tension [25], bending elasticity now becomes important [26,27]. It is possible, in principle, to determine equilibrium profiles by solving a field equation (the "shape equation") which follows from the minimization of the energy with respect to surface deformations. The forces and torques transmitted by the membrane are then imprinted on its shape.

The shape equation is a fourth-order nonlinear partial differential equation; as such, it can only be solved analytically in a few exceptional cases. One approach to sidestepping this obstacle is to expand the energy up to quadratic order. Unfortunately, this approach has the drawback that it is only valid if the surface is a weak perturbation of some underlying reference shape, such as a plane or a sphere. If the membrane is highly curved, linearization becomes inadequate and other strategies need to be developed.
In Ref. [9] an alternative approach was presented. It was shown that it is possible to relate the forces and torques to the local membrane geometry without any need to solve the shape equation itself. The link is formed by the surface stress tensor and its torque counterpart. This approach has already proven its value when applied to the problem of surfacemediated interactions [13-15]: particles bound to a membrane interact because they deform its shape [16-24]. In the traditional approach to this problem based on energy, the force between particles is calculated in three steps: first, the equilibrium shape of the membrane is determined for a given placement of the particles by solving the linearized shape equation. The energy of the surface is then evaluated by integration and finally differentiated with respect to appropriate placement variables of the bound particles. This approach relies heavily on the validity of the linearization. Instead, one can also relate the force between the particles directly to the local geometry of the surface via the surface stress tensor. It becomes possible to establish a host of valuable exact nonlinear results $[13,14]$.

In this context the particles were fixed by horizontal forces allowing all other degrees of freedom, among them their vertical positions or tilts, to equilibrate. To gain further insight a generalization is necessary which will be provided here: we will extend the framework developed in Refs. $[13,14]$ and explicitly address questions concerning the balance of torques. This will impose strong additional constraints on the shape of the membrane. To be concrete, we will examine symmetric two-particle configurations, thereby obtaining a transparent analytical expression describing the balance of torques which is valid in the fully nonlinear regime. We will examine exactly the interaction of two membrane-bound parallel cylinders. This is possible because the membrane profile behaves like a one-dimensional elasticum of Euler [28], a wormlike chain in the language of polymer physics [29-31]; thus, it can be integrated exactly. This apparently simple system displays remarkably subtle behavior. For strong membrane deformations the results of the small gradient approximation [21] fail and nonlinear effects, 
such as multiple solutions for one given set of parameters, emerge. Moreover, the simple exponential decay of the force or the cylinder tilt angle as a function of separation, which is correct for small deformations [21], gives way to a more complicated dependency.

The paper is organized as follows: in Sec. II we will discuss how torques on surface patches can be determined within the framework of Refs. $[9,10]$. To begin with, we will treat a geometrical Hamiltonian which is given by a surface integral over a local density. The behavior of this Hamiltonian under boundary rotations will be used to identify the torque tensor. This tensor will be written down explicitly for a fluid bilayer. Surface-mediated interactions between particles on an asymptotically flat membrane are considered in Sec. III. Total force and torque balance will be exploited to identify constraints on the geometry of the membrane. In Sec. IV the membrane geometry corresponding to two parallel cylinders will be determined exactly and the balance of forces and torques examined in detail. Finally, we summarize our main conclusions in Sec. V.

\section{TORQUES ON SURFACE PATCHES}

\section{A. Surface energetics}

Consider an interface which can be modeled as a twodimensional surface in three-dimensional Euclidean space. Such a surface $\Sigma$ is described locally by its position $\boldsymbol{X}\left(\xi^{1}, \xi^{2}\right) \in \mathbb{R}^{3}$, where the $\xi^{a}$ are any suitable set of local coordinates on the surface. The tangent vectors of $\Sigma$, $\boldsymbol{e}_{a}=\partial \boldsymbol{X} / \partial \xi^{a}=\partial_{a} \boldsymbol{X}(a, b \in\{1,2\})$, form a local coordinate frame; together with the extrinsic unit normal vector $\boldsymbol{n}=\boldsymbol{e}_{1} \times \boldsymbol{e}_{2} /\left|\boldsymbol{e}_{1} \times \boldsymbol{e}_{2}\right|$, they define two geometrical tensors on the surface: the metric $g_{a b}=\boldsymbol{e}_{a} \cdot \boldsymbol{e}_{b}$ and the extrinsic curvature $K_{a b}=\boldsymbol{e}_{a} \cdot \partial_{b} \boldsymbol{n}$ [32]. The trace of the extrinsic curvature $K=g_{a b} K^{a b}$ is the sum of the two principal curvatures whereas the Gaussian curvature $K_{\mathrm{G}}=\operatorname{det}\left(K_{a}^{b}\right)$ is their product. The covariant derivative on the surface will be denoted by $\nabla_{a}$, the corresponding Laplacian by $\Delta=\nabla_{a} \nabla^{a}$.

We associate with the surface an energy which can be described as a surface integral over a local density $\mathcal{H}$ :

$$
H_{\Sigma}[\boldsymbol{X}]=\int_{\Sigma} d A \mathcal{H}\left(g_{a b}, K_{a b}\right),
$$

where $d A=\sqrt{\operatorname{det}\left(g_{a b}\right)} d^{2} \xi=\sqrt{g} d^{2} \xi$ is the infinitesimal area element. The density $\mathcal{H}$ depends exclusively on surface scalars constructed using the metric and the extrinsic curvature tensor.

To determine the equilibrium shape of the surface the functional (1) is minimized with respect to deformations of $\Sigma$. These deformations are described by a change in the embedding functions $\boldsymbol{X} \rightarrow \boldsymbol{X}+\delta \boldsymbol{X}$. The corresponding variation of the Hamiltonian is [10]

$$
\delta H_{\Sigma}=\int_{\Sigma} d A \mathcal{E} \boldsymbol{n} \cdot \delta \boldsymbol{X}+\int_{\Sigma} d A \nabla_{a}\left[\mathcal{H}^{a b} \boldsymbol{e}_{b} \cdot \delta \boldsymbol{n}-f^{a} \cdot \delta \boldsymbol{X}\right] .
$$

Its bulk part is a surface integral over the Euler-Lagrange derivative $\mathcal{E}(\mathcal{H})$ times the normal projection of the surface

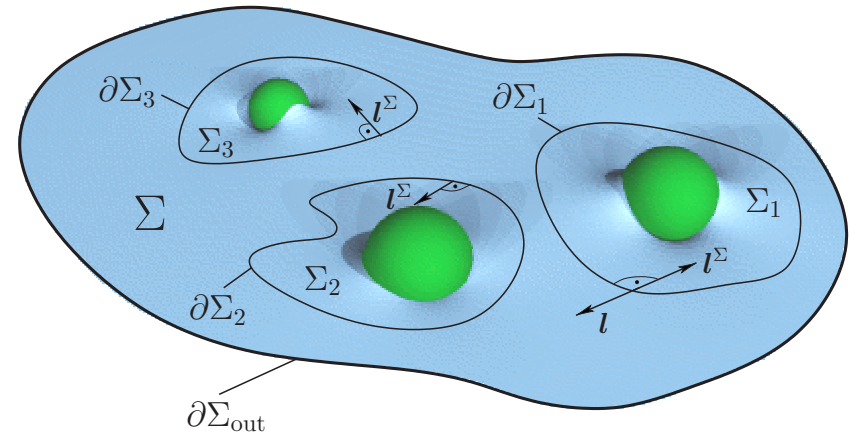

FIG. 1. (Color online) Surface patch $\Sigma$ with three disjoint boundary components $\partial \Sigma_{i}$ and an outer limiting boundary $\partial \Sigma_{\text {out }}$.

variation $\delta \boldsymbol{X}$. The second term is a surface integral over a divergence and can thus be recast as a boundary integral using the divergence theorem [33]. It involves two parts: a part proportional to $\delta \boldsymbol{X}$ identifies the surface stress tensor $\boldsymbol{f}^{a}$ $[9,10]$; the second part proportional to $\delta \boldsymbol{n}$ involves $\mathcal{H}^{a b}$ $=\partial \mathcal{H} / \partial K_{a b}$ and originates from the partial integration of second derivatives of the deformation. Note that additional terms would appear in the boundary term if $\mathcal{H}$ also depended on derivatives of $g_{a b}$ and $K_{a b}$.

According to Noether's theorem, every continuous symmetry of the Hamiltonian implies a conserved current. The one associated with the translational invariance of $H$ is the surface stress tensor $f^{a}$. Its conservation describes the balance of forces across curves drawn on the surface $[9,10]$. In an analogous way, the rotational invariance of $H$ implies the existence of another conserved tensor $\boldsymbol{m}^{a}$ which, as will be demonstrated in the next section, one can identify as the torque tensor.

\section{B. Identification of the torque tensor}

Consider a region of surface bounded by an outer limiting curve $\partial \Sigma_{\text {out }}$ and suppose that the bulk of this surface is in equilibrium with $N$ particles that are attached to it [14]. We now choose a curve around each of the particles, labeling these curves $\partial \Sigma_{i}, i=1, \ldots, N$. The outer curve $\partial \Sigma_{\text {out }}$ together with all $\partial \Sigma_{i}$ encloses the surface patch $\Sigma$ (see Fig. 1). Considering this patch first allows us to use variation (2) to identify the torque tensor as no particles are attached to $\Sigma$ and every point on it is free to equilibrate.

Under a constant infinitesimal rotation $\delta \boldsymbol{\alpha}$ of one of the boundaries $\partial \Sigma_{i}$, the position and normal vectors change according to $\delta \boldsymbol{X}=\delta \boldsymbol{\alpha} \times \boldsymbol{X}$ and $\delta \boldsymbol{n}=\delta \boldsymbol{\alpha} \times \boldsymbol{n}$. In this case, the boundary variation of the Hamiltonian (1) is equal to

$$
\begin{aligned}
\delta H_{\Sigma}^{(i)} & =-\delta \boldsymbol{\alpha} \cdot \oint_{\partial \Sigma_{i}} d s l_{a}^{\Sigma}\left[\boldsymbol{X} \times \boldsymbol{f}^{a}+\mathcal{H}^{a b} \boldsymbol{e}_{b} \times \boldsymbol{n}\right] \\
& =-\delta \boldsymbol{\alpha} \cdot \oint_{\partial \Sigma_{i}} d s l_{a}^{\Sigma} \boldsymbol{m}^{a}=-\delta \boldsymbol{\alpha} \cdot \boldsymbol{M}_{\Sigma, \mathrm{ext}}^{(i)}
\end{aligned}
$$

where the surface integral was converted into a boundary integral on the first line. The vector $\boldsymbol{l}^{\Sigma}=l_{a}^{\Sigma} \boldsymbol{e}^{a}$ is the unit vector which is normal to $\partial \Sigma_{i}$ and points out of the surface $\Sigma$; by 
construction, it is tangential to $\Sigma$ (see Fig. 1). The variable $s$ measures the arclength along $\partial \Sigma_{i}$.

In Eq. (3), the boundary integral is identified as the external torque $\boldsymbol{M}_{\Sigma}^{(i)}$ ext acting on $\Sigma$ via its boundary $\partial \Sigma_{i}$ : the corresponding change in energy is given by (minus) the scalar product of $\boldsymbol{M}_{\Sigma, \text { ext }}^{(i)}$ with the infinitesimal rotation angle $\delta \boldsymbol{\alpha}$. The external torque $\boldsymbol{M}_{\mathrm{ext}}^{(i)}$ on the surface patch $\boldsymbol{\Sigma}_{i}$ is then simply given by $-\boldsymbol{M}_{\Sigma}^{(i) \text { ext }}$ due to torque balance. Notice that this argument does not require us to assume that the EulerLagrange equation is satisfied on $\Sigma_{i}$.

To simplify the notation we will restrict our considerations to patch $\partial \Sigma_{1}$ without loss of generality and write $\boldsymbol{M}_{\mathrm{ext}}:=\boldsymbol{M}_{\mathrm{ext}}^{(1)}$, with

$$
\boldsymbol{M}_{\mathrm{ext}}=\oint_{\partial \Sigma_{1}} d s l_{a} \boldsymbol{m}^{a},
$$

where $\boldsymbol{l}=l_{a} \boldsymbol{e}^{a}=-\boldsymbol{l}^{\Sigma}$. The surface tensor

$$
\boldsymbol{m}^{a}=\boldsymbol{X} \times \boldsymbol{f}^{a}+\mathcal{H}^{a b} \boldsymbol{e}_{b} \times \boldsymbol{n}
$$

is the covariantly conserved torque tensor. It consists of two parts: a contribution due to the couple of the stress tensor $f^{a}$ about the origin as well as an intrinsic contribution proportional to $\mathcal{H}^{a b}$ which originates only from curvature terms. The value of the former does depend on the choice of origin; however, its intrinsic counterpart is independent of this choice. One can easily check that the divergence of $\boldsymbol{m}^{a}$, $\nabla_{a} \boldsymbol{m}^{a}$, is indeed zero in mechanical equilibrium as required by consistency with Noether's theorem.

The occurence of curvature terms in the Hamiltonian is ultimately due to the fact that the physical surface is not infinitely thin but has an inhomogeneous force distribution along its transverse direction [11]. If one captures this internal structure in the strictly two-dimensional Hamiltonian (1), the curvature terms give rise to an intrinsic torque density and a nonvanishing normal component of the stress tensor.

Note that the torque tensor, like the stress tensor, depends only on geometric properties of the surface (modulo a contribution that is due to a shift of the origin). It is thus always possible in principle to determine the torques operating on a patch of surface from a knowledge of the shape of the surface alone. The snag is to determine the correct shape.

It turns out, however, that exact geometric relations such as Eqs. (4) and (5) are still very useful, even when we lack details of the shape, if additional information-e.g., a symmetry-is available. This was shown in Refs. $[13,14]$ in the context of surface-mediated interactions between particles where the focus of interest was the force. To see how this works for torques, let us first write down Eq. (5) for the surface models we are interested in: soap films and fluid membranes.

\section{Special cases}

\section{Soap film}

The Hamiltonian density of a soap film is given by $\mathcal{H}=\sigma$, where $\sigma$ is the constant surface tension. This implies that $\mathcal{H}^{a b}=0$. Thus the intrinsic torque vanishes and, using $\boldsymbol{f}^{a}=-\sigma \boldsymbol{e}^{a}$, one obtains [9]

$$
\boldsymbol{m}^{a}=-\sigma\left(\boldsymbol{X} \times \boldsymbol{e}^{a}\right) .
$$

The torque on a surface patch $\Sigma_{1}$ is therefore given by

$$
\boldsymbol{M}_{\mathrm{ext}}=-\sigma \oint_{\partial \Sigma_{1}} d s(\boldsymbol{X} \times \boldsymbol{l}) .
$$

It is simple to interpret this expression: the tangential stress $-\sigma l$ provides a torque per unit length at every point of the contour $\partial \Sigma_{1}$. The line integral along $\partial \Sigma_{1}$ yields the total external torque on the surface patch.

\section{Fluid lipid membrane}

A symmetric fluid membrane can be described by the Hamiltonian [26,27]

$$
\mathcal{H}=\sigma+\frac{\kappa}{2} K^{2}+\bar{\kappa} K_{\mathrm{G}},
$$

where $\sigma$ is, as before, the surface tension, $\kappa$ the bending rigidity, and $\bar{\kappa}$ the saddle-splay modulus. Together the two constants $\sigma$ and $\kappa$ provide a characteristic length

$$
\lambda:=\sqrt{\frac{\kappa}{\sigma}}
$$

separating short length scales over which bending energy dominates from the large ones over which tension does.

The last term of the Hamiltonian density (8) can be written as the sum of a topological constant and a line integral over the geodesic curvature at the boundary of the membrane [32]. It does not contribute to the membrane stress tensor and does not enter the shape equation. However, it gives a contribution to the torque tensor [34]: the derivative of the density (8) with respect to $K_{a b}$ is

$$
\mathcal{H}^{a b}=\kappa K g^{a b}+\bar{\kappa}\left(K g^{a b}-K^{a b}\right) .
$$

Thus, the intrinsic torque does not vanish and, using the expression for $f^{a}$ from Ref. [14], the torque tensor can be written as

$$
\begin{aligned}
\boldsymbol{m}^{a}= & {\left[\kappa\left(K^{a b}-\frac{1}{2} K g^{a b}\right) K-\sigma g^{a b}\right]\left(\boldsymbol{X} \times \boldsymbol{e}_{b}\right)-\kappa\left(\nabla^{a} K\right)(\boldsymbol{X} \times \boldsymbol{n}) } \\
& +\left[(\kappa+\bar{\kappa}) K g^{a b}-\bar{\kappa} K^{a b}\right]\left(\boldsymbol{e}_{b} \times \boldsymbol{n}\right) .
\end{aligned}
$$

Inserting this result into Eq. (4) yields the external torque

$$
\begin{aligned}
\boldsymbol{M}_{\mathrm{ext}}= & \oint_{\partial \Sigma_{1}} d s\left\{\left[\frac{\kappa}{2}\left(K_{\perp}^{2}-K_{\|}^{2}\right)-\sigma\right](\boldsymbol{X} \times \boldsymbol{l})+\kappa K_{\perp \|} K(\boldsymbol{X} \times \boldsymbol{t})\right. \\
& \left.-\kappa\left(\nabla_{\perp} K\right)(\boldsymbol{X} \times \boldsymbol{n})-\kappa K \boldsymbol{t}\right\},
\end{aligned}
$$

on the membrane patch $\Sigma_{1}$. In expression (12) the unit tangent vector $\boldsymbol{t}=t^{a} \boldsymbol{e}_{a}=\boldsymbol{n} \times \boldsymbol{l}$ is introduced: it points along the integration contour $\partial \boldsymbol{\Sigma}_{1}$ and is perpendicular to $\boldsymbol{l}$ and $\boldsymbol{n}$. The projections of the extrinsic curvature onto the orthonormal basis of tangent vectors $\{\boldsymbol{l}, \boldsymbol{t}\}$ are given by $K_{\perp}=l^{a} l^{b} K_{a b}$, $K_{\|}=t^{a} t^{b} K_{a b}$, and $K_{\perp \|}=l^{a} t^{b} K_{a b}$. The symbol $\nabla_{\perp}=l_{a} \nabla^{a}$ denotes the directional derivative along the vector $\boldsymbol{l}$.

The intrinsic part of the torque tensor adds a contribution to the integrand in Eq. (12), which is proportional to the 
curvature $K$ and tangential to the contour of integration. Remarkably, the term $\boldsymbol{M}_{\mathrm{ext}}^{\bar{\kappa}}=\int_{\partial \Sigma_{1}} d s l_{a} \boldsymbol{m}_{\bar{\kappa}}^{a}$ originating from the Gaussian curvature vanishes. The reason for this is that its integrand can be written as a derivative with respect to the arclength $s$ :

$$
\begin{aligned}
l_{a} \boldsymbol{m}_{\bar{\kappa}}^{a} & =l_{a}\left[\bar{\kappa}\left(K g^{a b}-K^{a b}\right)\right]\left(\boldsymbol{e}_{b} \times \boldsymbol{n}\right)=-\bar{\kappa}\left(K_{\|} \boldsymbol{t}+K_{\perp \|} \boldsymbol{l}\right) \\
& =-\bar{\kappa}\left(t_{a} K^{a b} \boldsymbol{e}_{b}\right)=-\bar{\kappa} \nabla_{\|} \boldsymbol{n}=-\bar{\kappa} \frac{d \boldsymbol{n}}{d s},
\end{aligned}
$$

where the Weingarten equations $\nabla^{a} \boldsymbol{n}=K^{a b} \boldsymbol{e}_{b}$ were used and the symbol $\nabla_{\|}=t_{a} \nabla^{a}$ denotes the directional derivative along $t$-i.e. the derivative with respect to the arclength. Integrating $\nabla_{\|} \boldsymbol{n}$ over the closed contour $\partial \Sigma_{1}$ yields zero and thus $\boldsymbol{M}_{\mathrm{ext}}^{\bar{K}}=0$, even if an external torque is acting and even though the torque tensor comprises terms proportional to $\bar{\kappa}$. This result implies that torques on membrane patches do not depend on the last term of the Hamiltonian density (8) involving the Gaussian curvature $K_{\mathrm{G}}$. As shape and stresses are also independent of that term, we can neglect it in the following considerations.

In addition, if $K$ is constant on $\partial \Sigma_{1}$, the intrinsic part of the torque tensor does not contribute to $\boldsymbol{M}_{\text {ext }}$ at all. This is because the integral of $\boldsymbol{t}=d \boldsymbol{X} / d s$ vanishes along any closed contour.

It is straightforward to derive expressions analogous to Eq. (12) for any Hamiltonian of the form (1).

We will now show how the framework provided by the torque tensor may be applied to the study of membranemediated interactions.

\section{INTERFACE-MEDIATED INTERACTIONS BETWEEN PARTICLES ON ASYMPTOTICALLY FLAT INTERFACES}

Particles bound to an interface may interact via the deformations they impose on the surface geometry. Consider, for instance, a multiparticle configuration such as the one depicted in Fig. 1: forces as well as torques act on each of the particles due to the distortions in the shape caused by the others. Such a situation can thus only be stationary if the particle positions and orientations are constrained by external forces and torques, respectively. As we have shown, these forces and torques are determined completely by the surface geometry. The external torque acting on the particle that adheres to the surface patch $\Sigma_{1}$, for instance, is equal to the external torque $\boldsymbol{M}_{\text {ext }}$ acting on the whole patch as given by Eq. (4); this is because the torque tensor is divergence-free on any part of the surface not acted upon externally. The torque $\boldsymbol{M}^{(i)}$ we will consider in the following is the torque on particle $i$ mediated by the interface counteracting the corresponding external torque; the former is evidently equal to minus the latter (for $i=1$ one thus has $\boldsymbol{M}^{(1)}=-\boldsymbol{M}_{\mathrm{ext}}$ ). Similar arguments apply to the force $\boldsymbol{F}^{(i)}$ on the $i$ th particle as explained in detail in Refs. [13,14].

\section{A. Balance of forces and torques}

The total force and the total torque must vanish if the multiparticle configuration is in mechanical equilibrium:

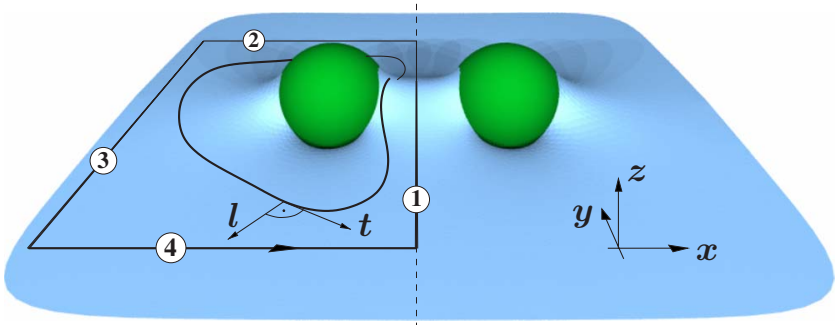

FIG. 2. (Color online) Two identical particles bound to an interface. As described in the text, it is possible to deform the contour of integration in order to exploit the available symmetries [14].

$$
\sum_{i=1}^{N} \boldsymbol{F}^{(i)}=\boldsymbol{F}_{\text {out }}, \quad \sum_{i=1}^{N} \boldsymbol{M}^{(i)}=\boldsymbol{M}_{\text {out }},
$$

where $\boldsymbol{F}_{\text {out }}$ is the force and $\boldsymbol{M}_{\text {out }}$ the torque on the outer boundary $\partial \Sigma_{\text {out }}$ (see again Fig. 1).

In the following, we will consider, in particular, a symmetric fluid membrane with nonvanishing surface tension $\sigma$ characterized by the Hamiltonian density (8). Its ground state in the absence of particles is an infinite flat plane with zero curvature. In this case the energy is proportional to the area and thus infinite. This infinite constant in the energy will play no role in the sequel, and we will recalibrate the energy to set it to zero.

Particles that are bound to the membrane typically deform its shape and increase its energy. These energy changes, however, must be finite whenever the forces and torques applied to fix the particle configuration are finite. This implies that the interface becomes asymptotically flat remote from the particles, even if $\sigma$ is infinitesimally small (see Appendix A).

It is useful to decompose forces and torques into a horizontal part parallel to the asymptotic plane and a vertical part orthogonal to it. When the surface tension vanishes, Kim et al. have shown that the vertical force and the horizontal torque on the outer boundary at infinity must vanish in equilibrium [23]. In Appendix A we extend their discussion to treat situations in which $\sigma \neq 0$. The change is nontrivial: it turns out that, whereas the vertical force vanishes as before, the horizontal torque does not necessarily vanish. In antisymmetric configurations, such as the ones that we will consider in the next section, it is this horizontal contribution on the outer boundary that makes it possible to balance an external torque.

In the following section, the complete set of equations describing force and total torque balance [see Eqs. (14)] in a symmetrical two-particle configuration will be derived.

\section{B. Two-particle configurations with symmetry}

Consider a symmetrical configuration of two identical particles bound to an asymptotically flat surface as described in Refs. $[13,14]$ (see Fig. 2). We label by $\{\boldsymbol{x}, \boldsymbol{y}, \boldsymbol{z}\}$ the Cartesian orthonormal basis vectors of three-dimensional space $\mathbb{R}^{3}$ adapted to the asymptotic plane. The vectors $\boldsymbol{x}$ and $\boldsymbol{y}$ lie parallel to this plane whereas $z$ is its upward pointing unit normal. 
We will discuss two possible symmetries: mirror symmetry in the $(y, z)$ plane (the symmetric case) or a twofold symmetry with respect to the $y$ axis (the antisymmetric case). The line joining corresponding points on the particles lies parallel to the $(x, z)$ plane. We place the origin of the coordinate system in the middle between the two particles on the intersection line of the asymptotic and symmetry plane (symmetric case) or the line of symmetry (antisymmetric case), respectively.

External torques on the surface can either be vertical or horizontal. The former cannot be compensated by the outer boundary. The physical explanation for this is that the surface does not resist shear forces. Horizontal torques, however, can be balanced (see Appendix A) which is necessary for a stable configuration: the separation between the particles is fixed by horizontal constraining forces. In the antisymmetric case, the two particles do not generally lie on the same line of action. Thus the forces will apply a horizontal torque $M_{y}$ to the surface which has to be balanced by the outer boundary. In the following, we will consider situations in which this torque is the only external torque on the entire surface.

This restriction does not exclude external vertical torques $\boldsymbol{M}^{(i)}$ on the individual particles; the symmetry will not be broken as long as all these torques cancel. Think, for instance, of a symmetric configuration consisting of two spheres on a soap film with a saddle-shaped (quadrupolar) line of contact [35]. A vertical torque $M_{z}$ on one of the particles is consistent with the symmetry as long as a torque $-M_{z}$ is applied to its partner. Such possibilities may, in principle, be accommodated within the formalism. Here, however, we will only consider situations in which the particle orientations have equilibrated and these torques vanish.

As explained previously, the torque on the left particle can be obtained by integrating the appropriate projection of the torque tensor along a contour surrounding the particle:

$$
\boldsymbol{M}=-\oint_{1-4} d s l_{a} \boldsymbol{m}^{a} .
$$

We choose the same contour of integration as used in Refs. $[13,14]$ (see Fig. 2): it consists of four branches with branch 1 lying on the symmetry plane/line and branches $2-4$ pulled open to infinity.

Using Eq. (14) together with the assumptions made above we conclude that

$$
\boldsymbol{M}=\boldsymbol{X}_{\mathrm{p}} \times \boldsymbol{F}=M \boldsymbol{y},
$$

where $\boldsymbol{F}=\boldsymbol{F} \boldsymbol{x}$ is the force on the left particle and $\boldsymbol{X}_{\mathrm{p}}$ is the position vector pointing from the fulcrum (i.e., the point about which the torque is acting) to the point where the force $\boldsymbol{F}$ is applied. We evaluate the torque about the origin in the Euclidean coordinate system $\{\boldsymbol{x}, \boldsymbol{y}, \boldsymbol{z}\}$. The torque on the outer boundary enclosing the particles is then given by $\boldsymbol{M}_{\text {out }}=2 \boldsymbol{M}$ in the antisymmetric case; it vanishes in the symmetric case.

The condition (16), together with the explicit expression for the torque $\boldsymbol{M}$ derived here as well as its counterpart for the force $\boldsymbol{F}$ derived in Refs. $[13,14]$, permits one to establish nonlinear relationships between geometrical quantities such as particle penetration or height differences for a given model.

\section{Equilibrium conditions from torque balance}

\section{General expressions for two finite-sized particles}

For a fluid membrane, the torque $\boldsymbol{M}$ on a particle can be obtained by inserting the corresponding torque tensor (11) into Eq. (15). As it evidently has to cancel the external torque on the particle, it can also be read off directly from the righthand side of Eq. (12) with the replacement of $\partial \Sigma_{1}$ by the contour 1-4 and an additional minus sign out front.

The resulting expression can be simplified further if one takes into account that the surface is asymptotically flat and possesses a certain symmetry. For finite-sized particles it is shown in Appendix A that the curvature terms of the torque tensor do not contribute to the torque on the membrane at infinity; the only term in the expression for the torque due to bending stems from branch 1 . Furthermore, the term proportional to $(\boldsymbol{X} \times \boldsymbol{t})$ vanishes for the two symmetries because $K_{\perp \|} K=0$ on branch 1 [14]. The torque $\boldsymbol{M}$ on the left particle is thus given by

$$
\begin{aligned}
\boldsymbol{M}= & \sigma \oint_{1-4} d s(\boldsymbol{X} \times \boldsymbol{l})-\kappa \int_{1} d s\left[\frac{1}{2}\left(K_{\perp}^{2}-K_{\|}^{2}\right)(\boldsymbol{X} \times \boldsymbol{l})\right. \\
& \left.-\left(\nabla_{\perp} K\right)(\boldsymbol{X} \times \boldsymbol{n})-K \boldsymbol{t}\right] \\
= & \sigma \oint_{2-4} d s(\boldsymbol{X} \times \boldsymbol{l})-\kappa \int_{1} d s[\boldsymbol{X} \times \boldsymbol{f}-K \boldsymbol{t}],
\end{aligned}
$$

where $\boldsymbol{f}=\left[\frac{1}{2}\left(K_{\perp}^{2}-K_{\|}^{2}\right)-\lambda^{-2}\right] \boldsymbol{l}-\left(\nabla_{\perp} K\right) \boldsymbol{n}$.

One now might naively expect that, in analogy to the curvature terms, the term due to surface tension vanishes along branches 2-4: the membrane becomes asymptotically flat at infinity and the corresponding force has no vertical component. As $\boldsymbol{X}$ lies in the asymptotic plane due to our choice of origin, it would appear to be self-evident that the line integral vanishes.

If this argument were correct, it would also apply to the complete outer boundary enclosing both particles and it would be impossible to balance the external torque. How then can a stable equilibrium be attained in the antisymmetric case if, as was argued earlier, an external torque is unavoidable?

Fortunately, a closer inspection of the integral shows that it does not necessarily always vanish: for when branches $2-4$ are sent to infinity, while the vertical force component indeed does converge to zero, the length $|\boldsymbol{X}|$ of the corresponding lever arm goes to infinity simultaneously. Additionally, one integrates along a contour whose length depends linearly on $|\boldsymbol{X}|$. These two effects permit a finite value to remain: if we parametrize the remote regions of the membrane in terms of a height function above the asymptotic plane and consider its series expansion (A5) for large $|\boldsymbol{X}|$, a nonvanishing horizontal torque remains if the slowest decaying term is of order $|\boldsymbol{X}|^{-1}$. In fact, from Eq. (A5) we obtain for the height function 


$$
\begin{aligned}
h(|\boldsymbol{X}|, \varphi)= & \left(C_{1} \cos \varphi+D_{1} \sin \varphi\right)|\boldsymbol{X}|^{-1} \\
& + \text { faster decaying terms }
\end{aligned}
$$

where $C_{1}$ and $D_{1}$ are constants and $\varphi$ is the azimuthal angle measured from the $x$ axis. Thus, branches 2-4 can contribute a torque if either of the two constants in Eq. (18) does not vanish. A closer inspection of the line integral along branches $2-4$ reveals that the term with coefficient $C_{1}$ contributes to a torque about the $y$ axis whereas the one including $D_{1}$ does so about $\boldsymbol{x}$ (cf. calculation in Appendix A for $\boldsymbol{M}_{\text {out }}$ ). The latter coefficient has to be equal to zero as we require the $x$ component of the torque $\boldsymbol{M}_{\text {out }}$ at the complete outer boundary to vanish (see again Appendix A).

Bearing these considerations in mind, one obtains the following important relations by combining Eqs. (16) and (17):

$$
\begin{aligned}
\boldsymbol{x} \cdot \boldsymbol{M} & =-\kappa \int_{1} d s[\boldsymbol{X} \cdot(\boldsymbol{f} \times \boldsymbol{x})]=0, \\
\boldsymbol{y} \cdot \boldsymbol{M} & =\sigma \oint_{2-4} d s[\boldsymbol{X} \cdot(\boldsymbol{l} \times \boldsymbol{y})]-\kappa \int_{1} d s[\boldsymbol{X} \cdot(\boldsymbol{f} \times \boldsymbol{y})-K(\boldsymbol{y} \cdot \boldsymbol{t})] \\
& =F\left(\boldsymbol{z} \cdot \boldsymbol{X}_{\mathrm{p}}\right), \\
\boldsymbol{z} \cdot \boldsymbol{M} & =-\kappa \int_{1} d s[\boldsymbol{X} \cdot(\boldsymbol{f} \times \boldsymbol{z})-K(\boldsymbol{z} \cdot \boldsymbol{t})]=0,
\end{aligned}
$$

where $F=-\kappa \int_{1} d s(\boldsymbol{f} \cdot \boldsymbol{x})-\sigma \int_{3} d s$ [14]. These are the expressions referred to at the end of the previous section. They place strong constraints on the geometry of the membrane and can be simplified further for the two different symmetries.

a. Symmetric case. In the symmetric case, the derivative of $K$ in the direction of $l$ along branch $1, \nabla_{\perp} K$, is zero. The curvature tensor is diagonal in $(\boldsymbol{l}, \boldsymbol{t})$ coordinates and thus $K_{\perp \|}$ vanishes. This implies that $K_{\|} t$ is equal to $\nabla_{\|} \boldsymbol{n}$ [cf. Eq. (13)] and $\int_{1} d s K|\boldsymbol{t}=\boldsymbol{n}|_{y=\infty}-\left.\boldsymbol{n}\right|_{y=-\infty}=0$.

Furthermore, branches $2-4$ do not provide a torque: the term in the height function (18) that could give rise to a torque about the $y$ axis is forbidden by the symmetry; i.e., its constant coefficient $C_{1}=0$. Thus,

$$
\sigma \oint_{2-4} d s(\boldsymbol{X} \times \boldsymbol{l})=0
$$

and Eq. (17) simplifies to the expression

$$
\boldsymbol{M}_{\mathrm{sym}}=-\kappa \int_{1} d s\left\{\left[\frac{1}{2}\left(K_{\perp}^{2}-K_{\|}^{2}\right)-\lambda^{-2}\right](\boldsymbol{X} \times \boldsymbol{x})-K_{\perp} \boldsymbol{t}\right\},
$$

which depends only on geometrical properties of the membrane at the symmetry plane. The force acting at each point along the midline is horizontal, and Eq. (19a) is fulfilled identically. The two relations (19b) and (19c) turn into

$$
-\kappa \int_{1} d s\left[f_{\mathrm{sym}}(z \cdot \boldsymbol{X})-K_{\perp}(\boldsymbol{y} \cdot \boldsymbol{t})\right]=F_{\mathrm{sym}}\left(z \cdot \boldsymbol{X}_{\mathrm{p}}\right),
$$

$$
\kappa \int_{1} d s\left[f_{\mathrm{sym}}(\boldsymbol{y} \cdot \boldsymbol{X})+K_{\perp}(\boldsymbol{z} \cdot \boldsymbol{t})\right]=0,
$$

with $f_{\text {sym }}=\left[\frac{1}{2}\left(K_{\perp}^{2}-K_{\|}^{2}\right)-\lambda^{-2}\right]$ and $F_{\text {sym }}=\sigma \Delta L-\frac{\kappa}{2} \int_{1} d s\left(K_{\perp}^{2}\right.$ $\left.-K_{\|}^{2}\right)$ where $\Delta L$ is the excess length of branch 1 compared to branch 3 [14].

b. Antisymmetric case. In the antisymmetric case branch 1 is a straight line and $K_{\perp}=K_{\|}=0$. The torque is now given by

$$
\boldsymbol{M}_{\text {antisym }}=\sigma \oint_{1-4} d s(\boldsymbol{X} \times \boldsymbol{l})+\kappa \int_{1} d s\left[\left(\nabla_{\perp} K\right)(\boldsymbol{X} \times \boldsymbol{n})\right] .
$$

In contrast to the symmetric case, the torque does not possess an internal part because the curvature $K$ vanishes everywhere along the contour. The force acting at each point along the midline is perpendicular to the $y$ axis. As the origin lies on this line the only components of the torque transmitted through branch 1 which are potentially nonvanishing are the $x$ and $z$ components. We have chosen, however, to set these components to zero [see the previous section and Eqs. (19a) and (19c)]. Thus, the contribution from branch 1 vanishes,

$$
\sigma \int_{1} d s(\boldsymbol{X} \times \boldsymbol{l})+\kappa \int_{1} d s\left[\left(\nabla_{\perp} K\right)(\boldsymbol{X} \times \boldsymbol{n})\right]=0,
$$

and the torque can be written as an integral along the boundaries 2-4:

$$
\boldsymbol{M}_{\text {antisym }}=\sigma \int_{2-4} d s(\boldsymbol{X} \times \boldsymbol{l})=F_{\text {antisym }}\left(\boldsymbol{z} \cdot \boldsymbol{X}_{\mathrm{p}}\right) \boldsymbol{y},
$$

where $F_{\text {antisym }}=\kappa \int_{1} d s\left[\lambda^{-2}(\boldsymbol{x} \cdot \boldsymbol{l}-1)+\left(\nabla_{\perp} K\right)(\boldsymbol{x} \cdot \boldsymbol{n})\right][14]$.

This equation implies a strong constraint on the asymptotics of the membrane. Far from the particles the term of leading order in the height function (18) has to be proportional to $|\boldsymbol{X}|^{-1}$. Otherwise, the external torque would not be balanced. The coefficient $C_{1}$ has to be finite, its value fixed by the external force responsible for the torque.

If the height function of the membrane is known, $\boldsymbol{M}_{\text {antisym }}$ can be easily calculated: in Appendix A we have derived expression (A7) for $\boldsymbol{M}_{\text {out }}$ in terms of the coefficients of the height function (A5)/(18). From this expression we obtain $\boldsymbol{M}_{\text {antisym }}=(1 / 2) \boldsymbol{M}_{\text {out }}=\pi \sigma C_{1} \boldsymbol{y}$, thus fixing $C_{1}=M_{\text {out }} /(2 \pi \sigma)$.

\section{Two infinitely long cylinders}

The situation changes if we consider two infinitely long cylinders of radius $R$ parallel to the $y$ axis that are separated by a distance $d$ (see Fig. 3). The size of the particles is no longer finite. Thus, it is necessary to exercise a little care when carrying over expressions derived for finite-sized particles.

a. Symmetric case. The symmetric case corresponds to two cylinders adhering to one side of the membrane. The curvature parallel to branch $1, K_{\|}$, vanishes as branch 1 degenerates into a line. The contributions from branch 2 and 4 cancel. No torque is exerted from branch 3: in contrast to the configurations with two finite-sized particles, the shape equation does not admit a term proportional to $|\boldsymbol{X}|^{-1}$ in the height function (see Sec. IV). 


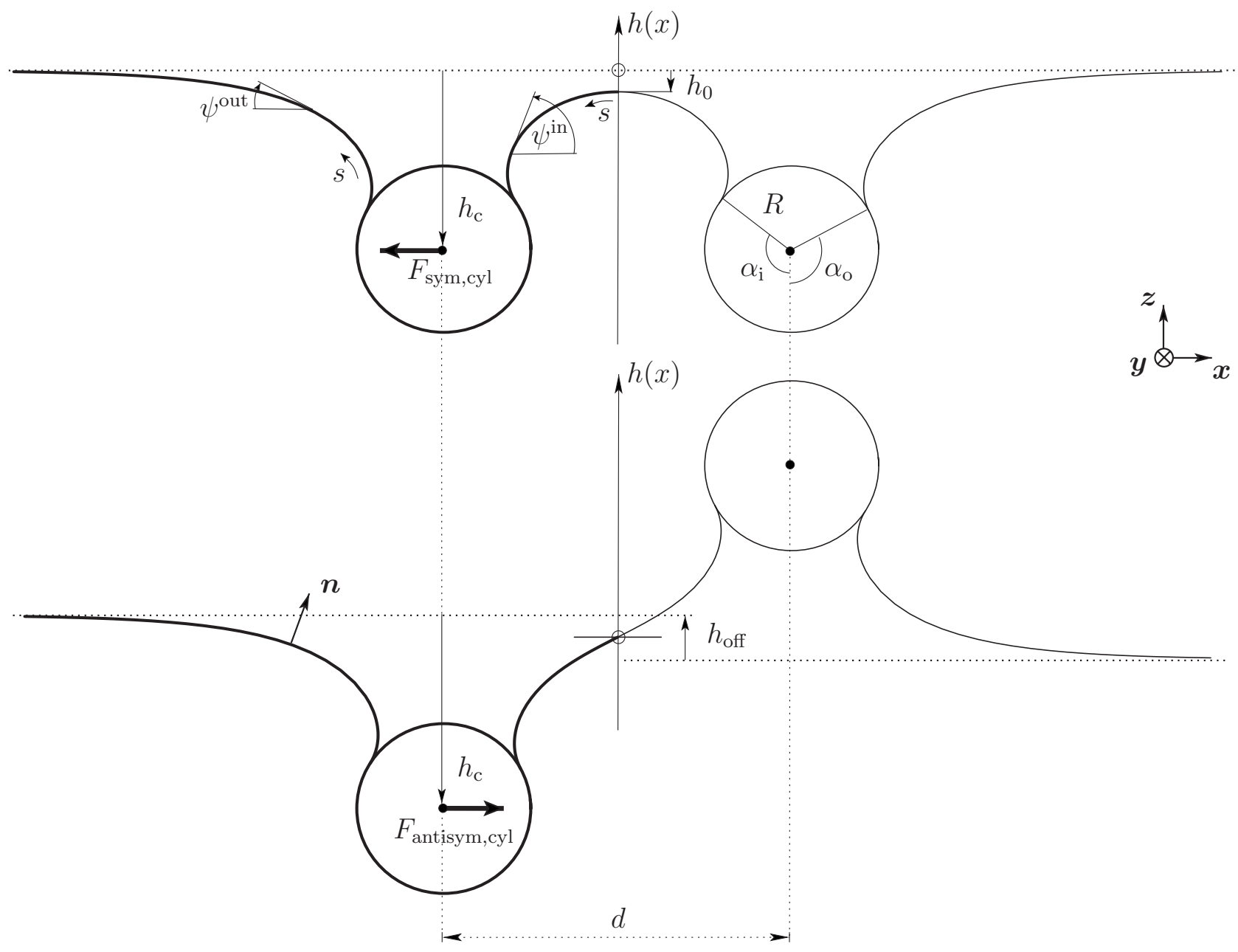

FIG. 3. Two parallel cylinders on a fluid membrane (symmetric and antisymmetric cases). The profiles are determined as explained in Sec. IV using the following parameters: $\alpha_{\mathrm{c}}=\alpha_{\mathrm{i}}+\alpha_{\mathrm{o}}=240^{\circ}, d=4, R=1$, and $\lambda=1$. The point about which the torques are evaluated is marked with ${ }^{\circ}$.

The torque per length $L$ of the cylinder with respect to the origin of the Euclidean coordinate system $\{\boldsymbol{x}, \boldsymbol{y}, \boldsymbol{z}\}$ is thus given by

$$
M_{\text {sym,cyl }} / L=\left(\frac{\kappa}{2} K_{\perp}^{2}-\sigma\right) h_{0}+\kappa K_{\perp},
$$

where the length $h_{0}$ is the distance between the origin and the midpoint of the profile (see Fig. 3). It is positive if this point lies below the origin.

Let $h_{\mathrm{c}}:=-\left(\boldsymbol{z} \cdot \boldsymbol{X}_{\mathrm{p}}\right)$ be the vertical distance between the center of the cylinder and the asymptotic plane (see again Fig. $3)$. It is positive if the two cylinders are located below the asymptotic plane.

Torque balance establishes a relationship between $h_{0}$ and $h_{\mathrm{c}}$, involving only the geometry on the midline, $M_{\mathrm{sym}, \mathrm{cyl}}$, given by Eq. (27): $M_{\text {sym,cyl }} \stackrel{(22)}{=}-h_{\mathrm{c}} F_{\text {sym,cyl }}$ where $F_{\text {sym,cyl }} / L$ $=-\frac{\kappa}{2} K_{\perp}^{2}[14]$ implies

$$
h_{0}=\frac{\kappa K_{\perp}-\frac{\kappa}{2} K_{\perp}^{2} h_{\mathrm{c}}}{\sigma-\frac{\kappa}{2} K_{\perp}^{2}}=\frac{K_{\perp}-\frac{1}{2} K_{\perp}^{2} h_{\mathrm{c}}}{\lambda^{-2}-\frac{1}{2} K_{\perp}^{2}} .
$$

Equation (28) remains valid if the interface is a soap film. The bending rigidity $\kappa$ then equals zero, and we immediately obtain the result that $h_{0}=0$. This is confirmed by the observation that the interface is flat everywhere and the forces on the left and right cylinders vanish [14].

If we parametrize the profile as a height $h(x)$ above the asymptotic reference plane, the curvature $K_{\perp}$ at branch 1 is exactly equal to $-h^{\prime \prime}(0)$ in the symmetric geometry, where dashes denote derivatives with respect to $x$. In Ref. [21] the height function $h(x)$ was determined at the linearized level in the small-gradient regime. Inserting it into Eq. (28) validates this relation within the accuracy of the linear approximation (see Appendix B).

b. Antisymmetric case. In the antisymmetric case the two cylinders adhere to opposite sides of the membrane. The torque on the left cylinder is determined as follows: at branch 1 , the line integral is zero. The contributions from branches 2 and 4 cancel as before. Thus, the torque at branch 3 had better not vanish if the horizontal torque due the force $F$ is to be balanced. However, the vertical force decreases faster than $1 / x$ as $x \rightarrow \pm \infty$ and thus cannot provide such a torque (cf. previous paragraph and Sec. IV). How can torques balance under these circumstances? 
The solution to this apparent contradiction is the following: the asymptotic plane for positive values of $x$ does not necessarily coincide with the one for negative values. This is because the corresponding sections of the profile are disconnected. It becomes possible for the membrane to shift vertically with an offset $h_{\text {off }}$ at the origin with respect to the asymptotic plane(s) (see Fig. 3). The torque is then simply given by

$$
M_{\text {antisym,cyl }} / L=-\frac{h_{\text {off }}}{2} \sigma .
$$

This offset may be related to $h_{\mathrm{c}}$ as follows. One has $M_{\text {antisym,cyl }} \stackrel{(26)}{=}-\left(h_{\mathrm{c}}-\frac{h_{\text {off }}}{2}\right) F_{\text {antisym,cyl }} \quad$ where $\quad F_{\text {antisym,cyl }} / L$ $=\sqrt{\sigma^{2}+\left(\kappa \nabla_{\perp} K_{\perp}\right)^{2}}-\sigma[14]$. One obtains

$$
\frac{h_{\text {off }}}{2 h_{\mathrm{c}}}=1-\frac{1}{\sqrt{1+\lambda^{4}\left(\nabla_{\perp} K_{\perp}\right)^{2}}},
$$

where $h_{\mathrm{c}}:=\left[-\left(z \cdot \boldsymbol{X}_{\mathrm{p}}\right)+h_{\text {off }} / 2\right]$ is now measured from the left asymptotic plane to the center of the cylinder (see again Fig. 3).

Expanding the inverse square root in Eq. (30) up to zeroth and second order, respectively, yields a lower and an upper bound on the ratio of $h_{\text {off }}$ to $h_{\mathrm{c}}$ :

$$
0 \leq \frac{h_{\text {off }}}{h_{\mathrm{c}}} \leq \lambda^{4}\left(\nabla_{\perp} K_{\perp}\right)^{2} .
$$

Obviously, the offset $h_{\text {off }}$ and $h_{\mathrm{c}}$ have the same sign. If $h_{\mathrm{c}}>0$, the left asymptotic plane lies above the right one as depicted in Fig. 3; for $h_{\mathrm{c}}<0$, the situation is reversed.

By setting $\kappa=0$, we can again provide a check for consistency. The offset is zero as in the symmetric case. This is in agreement with the result from Ref. [14] that the interface is flat and no force acts on the cylinders.

In Ref. [21] the offset is set to zero in the ansatz for the height function. On first inspection, this may appear to be an error. However, it turns out to be consistent if Eq. (30) is written in the small-gradient regime: the first nonvanishing term is of second order in the smallness parameter. As the height function of [21] is itself correct only to first order, the two results agree at the level of the approximation.

In this section several analytical conditions have been derived which link different geometric properties of the interface profile to each other. For the antisymmetric case we have seen that the external torque is compensated either by a vertical force component (if the particles are finite) or by an offset (if the particles are infinitely long cylinders). In the final part of this paper we will demonstrate the value of the framework developed in this section by showing how it can be applied to determine the exact shape of the membrane with two adhering cylinders.

\section{TWO CYLINDERS ON A FLUID MEMBRANE: EXACT SOLUTION}

\section{A. Determining the profile}

\section{Shape equation}

If two parallel cylinders adhere to the membrane, the profile can be decomposed into the following parts: two bound sections in which the cylinder and membrane are in contact, an inner section between the cylinders, and two outer sections that become flat for $x \rightarrow \pm \infty$ (see Fig. 3). The shape of the bound parts is determined by the geometry of the attached particle-i.e., a circular arc; the profiles of the free membrane sections are determined by solving a nonlinear differential equation. Solving the equation itself is simple; the subtlety, as we will see, is in the implementation of the boundary conditions.

We will introduce the "angle-arclength" parametrization of the profile: the angle $\psi(s)$ between the $x$ axis and the tangent to the profile as a function of arclength $s$ completely describes the shape of the membrane (see Fig. 3). It is connected to the curvature $K$ via the relationship $K=-\dot{\psi}$, where the overdot denotes a derivative with respect to $s$.

The nonlinear shape equation that determines the profile of the free membrane is given by $[8,36]$

$$
2 \ddot{K}+K^{3}-2 \lambda^{-2} K=0 .
$$

It possesses the first integral

$$
\dot{K}^{2}+\frac{1}{4} K^{4}-\lambda^{-2} K^{2}=E,
$$

where $E$ is a constant of integration.

It is straightforward to integrate Eq. (33) after a separation of variables to determine $s$ as a function of $K$ which can be inverted to express $K$ as a function of $s$. An additional integration yields the shape of the membrane, $\psi(s)$. One can do better, however, by taking advantage of the fact that the force $\boldsymbol{F}_{\text {mem }}$ per length $L$ of the cylinder at every point of the membrane is not only constant but also horizontal on each membrane section. Thus,

$$
\boldsymbol{F}_{\mathrm{mem}} / L=-l_{a} f^{a}=-\left(\frac{1}{2} \kappa K^{2}-\sigma\right) \boldsymbol{l}+\kappa \dot{K} \boldsymbol{n}=f_{\mathrm{mem}} \boldsymbol{x} .
$$

Projecting this equation onto $\boldsymbol{n}$ yields, with $\eta=f_{\text {mem }} / \sigma$ =const,

$$
\lambda^{2} \ddot{\psi}-\eta \sin \psi=0,
$$

where we have used $\boldsymbol{x} \cdot \boldsymbol{n}=-\sin \psi$ and $K=-\dot{\psi}$. Equation (35) appears in a number of other physical applications which include the motion of a simple pendulum [37], the shape of a fluid meniscus under gravity [38], or the behavior of elastic rods (Euler elastica) [28]. Despite appearances, it is completely equivalent to Eq. (33): both are of the same order in derivatives of $\psi$; furthermore, as we will show, the constant of integration $E$ can be written in terms of the scaled force $\eta$ and vice versa. Equation (35), however, has the advantage that it possesses a first integral with an integration constant 


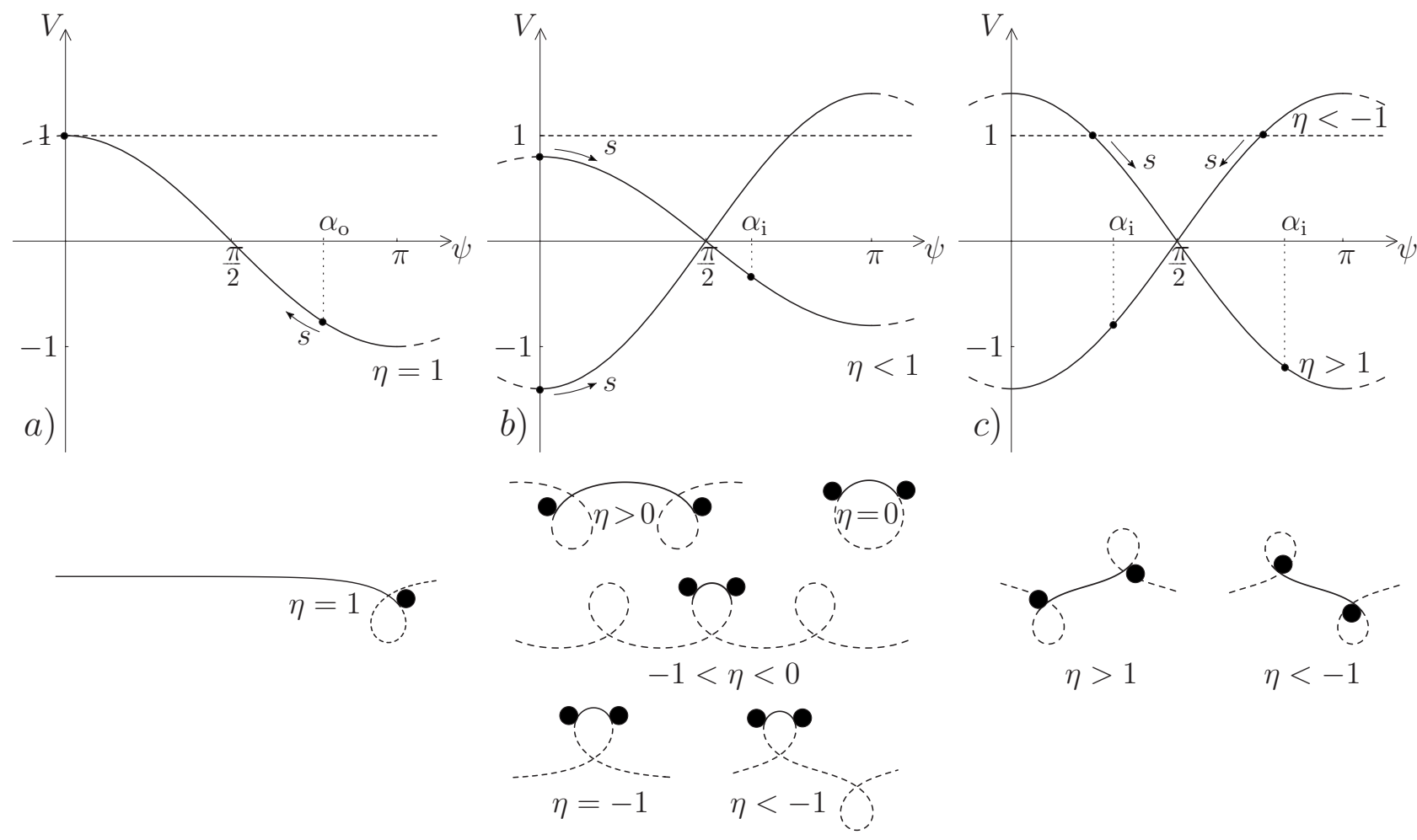

FIG. 4. Above: fictitious particle moving in the potential $V(\psi)=\eta \cos \psi$. (a) Outer section, (b) inner section, symmetric case, and (c) inner section, antisymmetric case. Below: corresponding solutions (38), (40), and (42) of Eq. (36).

that can be deduced from Eq. (34): the projection of the latter onto $\boldsymbol{l}$ yields, with $\boldsymbol{x} \cdot \boldsymbol{l}=\cos \psi$,

$$
\frac{\lambda^{2}}{2} \dot{\psi}^{2}+\eta \cos \psi=1 \text {. }
$$

Equation (36) can be interpreted as an energy balance of a fictitious particle moving in the potential $V(\psi)=\eta \cos \psi$, with displacement variable $\psi$, mass $\lambda^{2}$, and energy 1 . The details of the "motion" depend on the value of $\eta$ (see below and Fig. 4). From Eq. (36) follows

$$
K= \pm \lambda^{-1} \sqrt{2(1-\eta \cos \psi)} .
$$

It is simple to check that $K$ satisfies the shape equation (32). The constants $\eta$ and $E$ are not independent; the relation between them, $\eta= \pm \sqrt{E \lambda^{4}+1}$, is found by substituting $K$ into Eq. (33).

In the following, we will determine the solutions of Eq. (36) for the free sections of the profile if the cylinders are separated by a distance $d$. Due to the symmetry, it is sufficient to consider only the left half of the membrane (where $x<0$; see Fig. 3). While the outer sections are qualitatively identical for the two symmetries, the inner sections differ. Equation (36) is a first-order differential equation involving one unknown constant $\eta$. Thus two boundary conditions are required for each section.

\section{Outer section}

The boundary conditions for the outer section are the following: the free profile leaves the cylinder at a fixed contact angle $\psi(0)=\alpha_{\mathrm{o}}$. We assume without loss of generality that $\alpha_{\mathrm{o}}>0$ and $\dot{\psi} \leq 0$ [39]. At infinity, both $\psi$ and $\dot{\psi}$ vanish. If we insert these two conditions into Eq. (36), we obtain $\eta=1$ on this section. This implies that $E=0$ everywhere along the profile. The corresponding potential $V(\psi)$ is plotted in Fig. 4(a): the solution with $\psi(0)=\alpha_{\mathrm{o}}$ decreases monotonically to $\psi=0$ (where $V=1$ ) as $s \rightarrow \infty$.

The corresponding shape equation can be solved in terms of elementary functions. One obtains

$$
\psi^{\text {out }}(\widetilde{s})=4 \arctan \left[\tan \frac{\alpha_{\mathrm{o}}}{4} e^{-\widetilde{s}}\right],
$$

where $\tilde{s}:=s / \lambda$. Incidentally, the function (38) also describes the shape of a straight fluid meniscus that approaches a solid surface at the angle $\alpha_{\mathrm{o}}[25,38]$.

The function (38) decays exponentially with increasing arclength. Thus, in contrast to the case of finite-sized particles, the horizontal torque due to a vertical force component, $|M| \approx \sigma|x| \sin \psi^{\text {out }} \approx \sigma|x| \psi^{\text {out }}$, vanishes at infinity (see Appendix A and Sec. III C).

\section{Inner section}

We first establish the connection between the total arclength of the inner profile, $2 s_{\text {mid }}$, and the contact angle between the cylinder and the inner membrane, $\alpha_{\mathrm{i}}$. If the arclength is measured from the midline, we have

$$
\frac{\tilde{d}}{2}-\widetilde{R} \sin \alpha_{\mathrm{i}}=\int_{0}^{\tilde{s}_{\text {mid }}} d \tilde{s} \cos \psi(\widetilde{s}),
$$

where all lengths, as before, are scaled with $\lambda$. This condition determines the value of $\widetilde{s}_{\text {mid }}$ implicitly in terms of $\alpha_{\mathrm{i}}$. 
We again consider only positive values of $\psi$. The solution for negative angles is obtained by reversing the sign of the functions (40) and (42) which corresponds to a reflection of the profile in the $(x, y)$ plane. Note also that $K=+\dot{\psi}$ for all shapes of the inner section due to the chosen orientation of the arclength.

a. Symmetric case. In the symmetric case, $\psi(0)$ is equal to zero. From Eq. (36) we thus obtain $\eta \leq 1$. For $\eta=1$, the cylinders are infinitely far apart and do not interact. If we omit this trivial case, the following solutions for different values of $\eta$ are obtained [28,31,40] [see Fig. 4(b)]:

$$
\psi_{\mathrm{sym}}^{\text {in }}(\widetilde{s})= \begin{cases}\pi+2 \operatorname{am}\left(\tilde{s} \sqrt{\frac{\eta}{m}}-K(m), m\right), & 0<\eta<1, \\ \sqrt{2} \widetilde{s}, & \eta=0, \\ 2 \operatorname{am}\left(\tilde{s} \sqrt{\frac{|\eta|}{m}}, m\right), & -1<\eta<0, \\ 4 \arctan \left[\tanh \left(\frac{\tilde{s}}{2}\right)\right], & \eta=-1, \\ \arccos \left[1-\frac{2}{m} \operatorname{sn}^{2}\left(\tilde{s} \sqrt{|\eta|}, \frac{1}{m}\right)\right], & \eta<-1,\end{cases}
$$

where $\operatorname{am}(s, m)$ is the Jacobi amplitude with parameter $m$ and $\operatorname{sn}(s, m)=\sin [\operatorname{am}(s, m)]$. The symbol $K(m)$ denotes the complete elliptic integral of the first kind [41]. The parameter $m$ is given by $m:=\frac{2|\eta|}{1+|\eta|} \in[0,2]$.

b. Antisymmetric case. If the two cylinders adhere antisymmetrically, $\dot{\psi}$ must vanish at the midline. Thus,

$$
\cos \psi_{\text {mid }}{ }^{(36)}=\frac{1}{\eta},
$$

where $\psi_{\text {mid }}=\psi(0)$ is the angle at the midline. The case where $\psi_{\text {mid }}$ equals zero or $180^{\circ}$ corresponds again to the trivial solution with the two cylinders infinitely far apart. No solution exists for $\psi_{\text {mid }}=90^{\circ}$ as the scaled force $\eta$ has to remain finite in an equilibrium situation. If $0<\psi_{\text {mid }}<90^{\circ}, \eta>1$, and, for $-\frac{1}{\sqrt{\eta}} K\left(\frac{1}{m}\right) \leq \tilde{s} \leq \frac{1}{\sqrt{\eta}} K\left(\frac{1}{m}\right)[28,31]$ [see Fig. 4(c)],

$$
\psi_{\text {antisym }}^{\text {in }}(\widetilde{s})=\arccos \left\{\frac{2}{m} \operatorname{sn}^{2}\left[\tilde{s} \sqrt{\eta}-K\left(\frac{1}{m}\right), \frac{1}{m}\right]-1\right\} ;
$$

if it is greater than $90^{\circ}$ and lower than $180^{\circ}, \eta<-1$ and, for $-\frac{1}{\sqrt{\eta}} K\left(\frac{1}{m}\right) \leq \widetilde{s} \leq \frac{1}{\sqrt{\eta}} K\left(\frac{1}{m}\right)$ [28] [see again Fig. 4(c)],

$$
\psi_{\text {antisym }}^{\text {in }}(\widetilde{s})=\arccos \left\{1-\frac{2}{m} \operatorname{sn}^{2}\left[\widetilde{s} \sqrt{|\eta|}-K\left(\frac{1}{m}\right), \frac{1}{m}\right]\right\} .
$$

The parameter $m$ is defined as above and varies now between 1 and 2.

\section{Boundary conditions at the cylinders}

To obtain the profile of the membrane for given separation $\widetilde{d}$ and cylinder radius $\widetilde{R}$, one has to determine the values of the scaled force $\eta$ and the contact angles $\alpha_{\mathrm{o}}$ and $\alpha_{\mathrm{i}}$. The value of $\eta$ for any given $\alpha_{\mathrm{i}}$ is determined implicitly by the requirement that

$$
\psi\left(\widetilde{s}_{\text {mid }}\right)=\alpha_{\mathrm{i}},
$$

where $\widetilde{s}_{\text {mid }}$ is itself implicitly given by condition (39); the values of $\alpha_{\mathrm{i}}$ and $\alpha_{\mathrm{o}}$ depend on the boundary conditions at the cylinder. We will discuss two possibilities here: we either fix the area of contact between the cylinders and the membrane or consider attachment due to a finite adhesion energy $w$ per area between the membrane and the cylinders.

a. Fixed area of contact. Suppose that the area of contact is fixed to

$$
\alpha_{\mathrm{c}}=\alpha_{\mathrm{o}}+\alpha_{\mathrm{i}}=\text { const. }
$$

Torque balance will fix the relative values of $\alpha_{\mathrm{o}}$ and $\alpha_{\mathrm{i}}$.

The torque about the cylinder axis has to vanish in equilibrium. This is the case if the total energy $E_{\text {tot }}\left(\alpha_{\mathrm{i}}\right)$ of the system exhibits a local extremum. The corresponding torque balance can be written as

$$
0=\widetilde{K}_{\mathrm{i}}-\widetilde{K}_{\mathrm{o}}-\widetilde{R}\left(\eta \cos \alpha_{\mathrm{i}}-\cos \alpha_{\mathrm{o}}\right)=\widetilde{K}_{\mathrm{i}}-\widetilde{K}_{\mathrm{o}}+\frac{\widetilde{R}}{2}\left(\widetilde{K}_{\mathrm{i}}^{2}-\widetilde{K}_{\mathrm{o}}^{2}\right),
$$

where $\widetilde{K}_{\mathrm{i}}:=\lambda K_{\mathrm{i}}$ and $\widetilde{K}_{\mathrm{o}}:=\lambda K_{\mathrm{o}}$. Equation (45) has two solutions $\widetilde{K}_{\mathrm{i}}=\widetilde{K}_{\mathrm{o}}$ and $\widetilde{K}_{\mathrm{i}}=-\widetilde{K}_{\mathrm{o}}-2 / \widetilde{R}$. The latter implies that either $\widetilde{K}_{\mathrm{i}}$ or $\widetilde{K}_{\mathrm{o}}$ is smaller than $-1 / \widetilde{R}$. Since the cylinder has a curvature of $-1 / \widetilde{R}$ in scaled units and the membrane and cylinder must not intersect, the second solution can be ruled out. The two contact curvatures must agree:

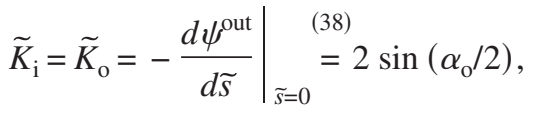

and

$$
\eta \cos \alpha_{\mathrm{i}}=\cos \alpha_{\mathrm{o}} .
$$

The values of $\eta, \alpha_{\mathrm{i}}, \alpha_{\mathrm{o}}$, and $\widetilde{s}_{\text {mid }}$ for given separation and cylinder radius can be determined numerically by solving the conditions (39), (43), (44), and (46) simultaneously. As an example, the two profiles for $\widetilde{d}=4, \widetilde{R}=1$, and $\alpha_{\mathrm{c}}=240^{\circ}$ are plotted in Fig. 3.

$b$. Adhesion balance. If the cylinders attach to the membrane due to a finite adhesion energy $w$, the contact curvature condition holds (see [42], Sec. 12, problem 6, [43], and [44]):

$$
\widetilde{K}_{\mathrm{i}}=\widetilde{K}_{\mathrm{o}}=-1 / \widetilde{R}+\sqrt{\widetilde{w}},
$$

where $\tilde{w}:=\frac{2 w \lambda^{2}}{\kappa}$. The curvatures are equal as in case $a$ and torque balance (45) is fulfilled automatically. The contact angle $\alpha_{\mathrm{o}}$ can be determined from Eq. (46). Finally, $\eta, \alpha_{\mathrm{i}}$, and $\widetilde{s}_{\text {mid }}$ can be calculated using Eqs. (39), (43), and (48).

The adhesion energy $\widetilde{w}$ and the wrapping angle $\alpha_{\mathrm{c}}$ are conjugate variables. Setting one of them to a constant value implies that the other will adjust in equilibrium. Questions of stability depend on which of the two variables is fixed: a 
profile found to be stable under constant $\alpha_{\mathrm{c}}$ is not necessarily stable under the constant $\widetilde{w}$. To avoid problems of this kind we will focus, in the following, on the constant wrapping angle scenario.

Note that the boundary condition (46) does not always fix a unique profile. The total energy $E_{\text {tot }}\left(\alpha_{\mathrm{i}}\right)$ of the system may possess more than one minimum; thus, profiles which are locally stable may exist. In the next subsection we will see that this is indeed the case for certain ranges of values of $\tilde{d}$, $\widetilde{R}$, and $\alpha_{\mathrm{c}}$.

\section{B. Conditions from torque balance}

Now that we possess the complete profile we can go back and reexamine Eqs. (28) and (30) and study the behavior of $\widetilde{h}_{0}:=h_{0} / \lambda$ and $\widetilde{h}_{\text {off }}:=h_{\text {off }} / \lambda$ as a function of distance $\widetilde{d}$ for different values of $\widetilde{R}$ and $\alpha_{\mathrm{c}}$.

a. Symmetric case. In the symmetric case, $\widetilde{K}_{\perp}=\sqrt{2(1-\eta)}$ and Eq. (28) can be written as

$$
\tilde{h}_{0}=\eta^{-1}\left[\sqrt{2(1-\eta)}-(1-\eta) \tilde{h}_{\mathrm{c}}\right],
$$

where

$$
\tilde{h}_{\mathrm{c}}=2 \sin \left(\alpha_{\mathrm{o}} / 2\right)-\widetilde{R} \cos \alpha_{\mathrm{o}} .
$$

The height $\widetilde{h}_{0}$ can be determined as a function of separation $\tilde{d}$ for fixed cylinder radius $\widetilde{R}$ and different wrapping angles $\alpha_{\mathrm{c}}$. If the value of $\alpha_{\mathrm{c}}$ is small, the solution coincides with the result of the small gradient approximation (B8) as expected. The value of $\tilde{h}_{0}$ falls off exponentially with the decay length $\ell:=2 \lambda$ for increasing separation $\tilde{d}$.

For higher angles $\alpha_{\mathrm{c}}$, however, the behavior changes at small values of $\tilde{d}$ due to the breakdown of the small gradient approximation (see Fig. 5 for $\widetilde{R}=1$ ): the energy $E_{\mathrm{tot}}\left(\alpha_{\mathrm{i}}\right)$ exhibits two minima instead of one [45]. These minima correspond to distinct stable profiles with different $\alpha_{\mathrm{i}}$ and $\widetilde{h}_{0}$. If $\alpha_{\mathrm{c}}=180^{\circ}$, they possess the same energy. If $\alpha_{\mathrm{c}}<180^{\circ}$, the global minimum is located at the smaller value of $\alpha_{\mathrm{i}}$ and the corresponding $\widetilde{h}_{0}$ is always positive; i.e., the midpoint lies below the reference plane (see again Fig. 5). For larger contact angles $\alpha_{\mathrm{c}}$ this behavior is reversed: the higher value of $\alpha_{\mathrm{i}}$ and the lower value of $\tilde{h}_{0}$ correspond to the global energy minimum. The midpoint may now even lie above the reference plane.

In Fig. 6 the tilt angle $\alpha_{\mathrm{t}}:=\frac{\alpha_{\mathrm{c}}}{2}-\alpha_{\mathrm{i}}$ is plotted as a function of distance $\widetilde{d}$ for $\widetilde{R}=1$. It decays exponentially with length $\lambda$ at large separations. If $\alpha_{\mathrm{c}}<180^{\circ}$, the tilt angle of the global energy minimum is always positive which corresponds to profiles such as the one plotted in Fig. 5(a). If $\alpha_{\mathrm{c}}>180^{\circ}$, the tilt angle of the global energy minimum is negative. For $\alpha_{\mathrm{c}}$ $=180^{\circ}$ and for all $\tilde{d} \gtrsim 3, \alpha_{\mathrm{t}}$ is equal to zero; at $\tilde{d} \approx 3$, the function bifurcates into two branches of the same energy. A closer inspection of Eq. (47) explains these findings: either $\alpha_{\mathrm{i}}$ is exactly $90^{\circ}$, and thus $\alpha_{\mathrm{t}}=0$, or $\eta=-1$. At the bifurcation point both conditions hold. For $\widetilde{d} \leqslant 3$ the profiles with

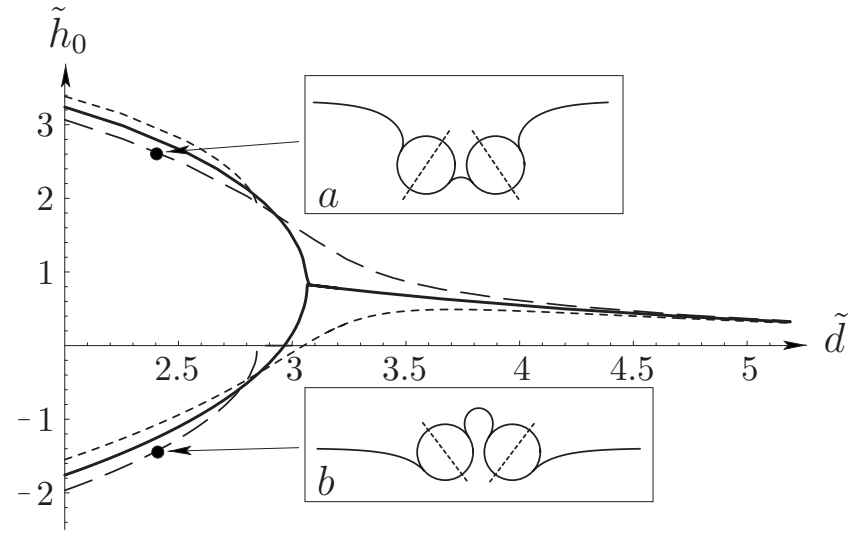

FIG. 5. The height $\tilde{h}_{0}$ at the midline as a function of separation $\tilde{d}$ for $\widetilde{R}=1$ and $\alpha_{\mathrm{c}}=170^{\circ}$ (long-dashed line), $180^{\circ}$ (bold solid line), and $190^{\circ}$ (short-dashed line). At $\widetilde{d}=2$ the two cylinders are in contact with each other and cannot draw nearer. For $\tilde{d} \lesssim 3$ two locally stable solutions can be found which correspond to different angles $\alpha_{\mathrm{i}}$. In the insets $a$ and $b$ the two solutions for $\tilde{d}=2.4$ and $\alpha_{\mathrm{c}}=170^{\circ}$ are plotted where $\alpha_{\mathrm{i}}=50.9^{\circ}$ (a) and $122.2^{\circ}$ (b), respectively. Profile (a) corresponds to the global energy minimum here.

$\eta=-1$ are local minima of same energy whereas $\alpha_{\mathrm{t}}=0$ corresponds to the intermediate maximum we do not discuss further here.

b. Antisymmetric case. From Eqs. (30) and (35) one obtains with $\nabla_{\perp} K_{\perp}=-\left.\ddot{\psi}\right|_{\tilde{s}=0}, K_{\perp}=0$, and $\eta>1$ :

$$
\frac{\widetilde{h}_{\text {off }}}{2 \widetilde{h}_{\mathrm{c}}}=1-\frac{1}{\eta},
$$

where $\tilde{h}_{\mathrm{c}}$ is given by Eq. (50).

If the two cylinders are closer than $2 \widetilde{R}$, one additionally has to check for every stable profile whether the particles overlap or not. To avoid complications of this kind we will only consider separations $\tilde{d} \geq 2 \tilde{R}$. In contrast to the symmet-

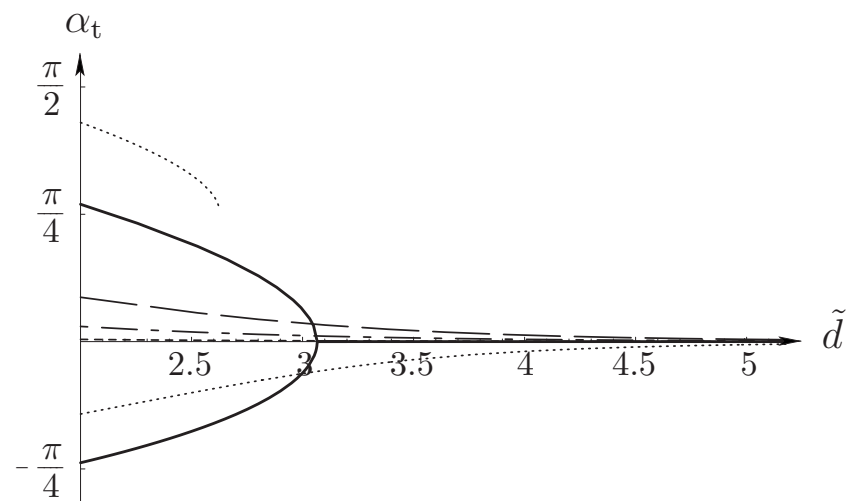

FIG. 6. The tilt angle $\alpha_{\mathrm{t}}$ (symmetric case) as a function of separation $\tilde{d}$ for $\tilde{R}=1$ and $\alpha_{\mathrm{c}}=10^{\circ}$ (short-dashed line), $45^{\circ}$ (dash-dotted line), $90^{\circ}$ (long-dashed line), $180^{\circ}$ (bold solid line), and $240^{\circ}$ (dotted line). 


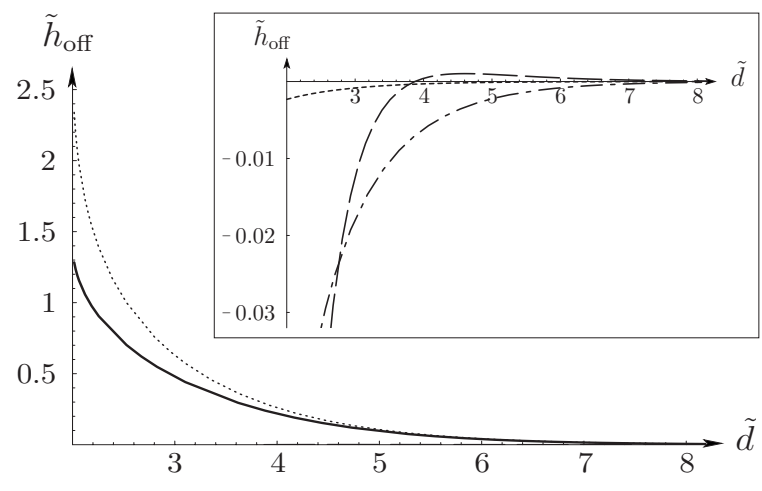

FIG. 7. The offset $\tilde{h}_{\text {off }}$ as a function of separation $\tilde{d}$ for $\widetilde{R}=1$ and different contact angles. The line styles are chosen as in Fig. 6.

ric case, one then finds a single solution for given $\widetilde{d}, \widetilde{R}$, and $\alpha_{\mathrm{c}}$.

In Fig. $7, \tilde{h}_{\text {off }}$ is plotted as a function of distance $\tilde{d}$. For small contact angles, $\tilde{h}_{\text {off }}$ is negative which implies that the asymptotic plane on the left lies below the one on the right and below the center of the left cylinder. For intermediate values of $\alpha_{\text {c }}$ (such as $90^{\circ}$ if $\widetilde{R}=1$ ) $\tilde{h}_{\text {off }}$ can be either positive or negative depending on the separation of the cylinders (see again Fig. 7). If $\alpha_{\mathrm{c}}$ is further increased, $\tilde{h}_{\text {off }}$ is positive for all separations and the profiles resemble the one which is plotted in the lower part of Fig. 3.

The tilt angle $\alpha_{\mathrm{t}}$ is always equal to zero for $\alpha_{\mathrm{c}}=180^{\circ}$. The solution $\eta=-1$ which also solves Eq. (47) is now forbidden due to the symmetry. For $\alpha_{\mathrm{c}}>180^{\circ}$ the tilt angle is positive; for $\alpha_{\mathrm{c}}<180^{\circ}$ it is negative. In both cases it decays exponentially with length $\lambda$.

\section{Forces between the cylinders}

Using the stress tensor $[13,14]$, the force on the left cylinder is given by

$$
F_{\text {cyl }} / L \stackrel{(34)}{=} f_{\text {mem }}-\sigma=\sigma(\eta-1) .
$$

Thus, one has only to determine the value of $\eta$; the force follows directly.

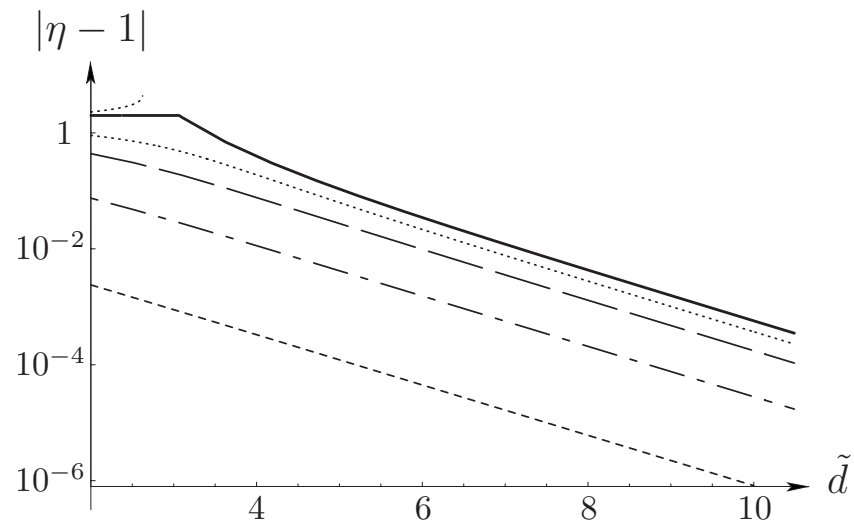

FIG. 8. Absolute value of the scaled force $|\eta-1|$ as a function of separation $\tilde{d}$ for $\widetilde{R}=1$ and different contact angles (symmetric case). The line styles are again chosen as in Fig. 6.

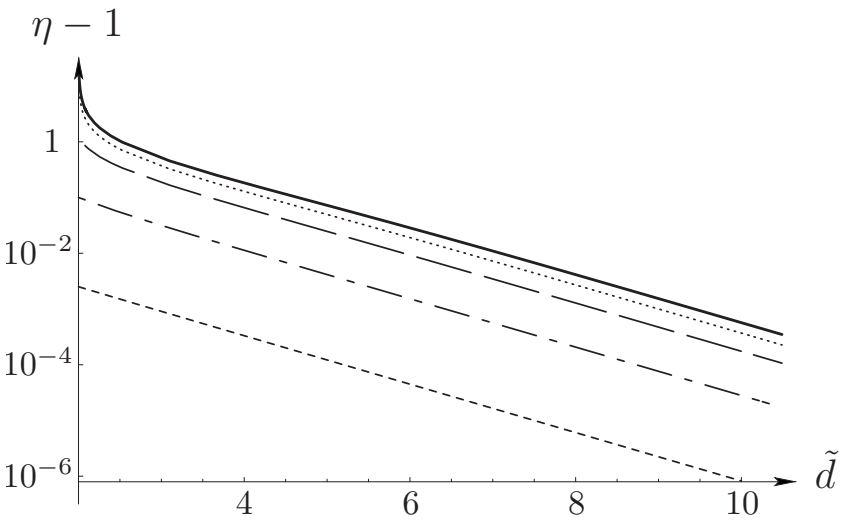

FIG. 9. Scaled force $\eta-1$ as a function of separation $\tilde{d}$ for $\widetilde{R}=1$ and different contact angles (antisymmetric case). The line styles are chosen as in Fig. 6.

In Figs. 8 and 9 the absolute value of the scaled force, $|\eta-1|$, is plotted for the symmetric and antisymmetric cases. At large separations it decays exponentially as predicted by the linearized theory [21]. The underlying small gradient approximation, however, breaks down if the curvatures along the profile become too high-i.e., for large contact area and small separation. In Fig. 10 the exact solutions are compared with the small gradient approximation for the symmetric geometry by plotting $|\eta-1|$ as a function of $\alpha_{\mathrm{c}}$ for $\tilde{d}=3$ and $\widetilde{R}=1$. The approximate solution increasingly underestimates the force as the contact angle is increased.

As soon as the small gradient approximation breaks down, different features appear: in the antisymmetric case, $|\eta-1|$ increases faster for decreasing separation than a small gradient approximation would anticipate. In the symmetric case, two solutions can be found (cf. previous subsection). The global energy minimum typically corresponds to the solution with the smaller absolute value of the force. Remarkably, the force is constant $\left[F_{\text {cyl }} /(L \sigma)=-2\right]$ for $\alpha_{\mathrm{c}}=180^{\circ}$ and $\tilde{d} \lesssim 3$.

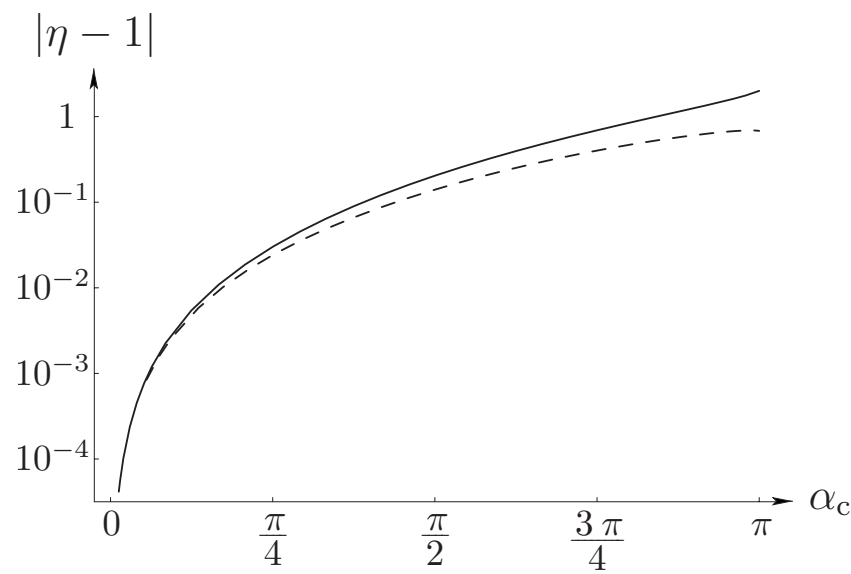

FIG. 10. $|\eta-1|$ as a function of $\alpha_{\mathrm{c}}$ for $\tilde{d}=3$ and $\widetilde{R}=1$ in the symmetric case. The small gradient approximation is plotted with a dashed line, the numerically exact solution with a solid line. 


\section{CONCLUSIONS}

We have extended the formalism which was originally developed in Refs. [9,10] and primarily applied to forces $[13,14]$ to study torques on surface patches. The underlying torque tensor depends only on geometric properties of the surface (modulo the contribution that is due to a shift of the origin). Therefore, it is possible to relate torques on particles which are bound to an interface directly to the interface geometry. For the case of the Helfrich Hamiltonian we have explicitly worked out the torque tensor. Remarkably, its contribution from the Gaussian curvature part, even though nonzero, vanishes upon closed-loop integration and therefore does not transmit torques between particles.

By exploiting torque and force balance in the context of membrane-mediated interactions, several analytical conditions on the interface geometry have been derived for twoparticle configurations obeying a certain symmetry. In the symmetric two-particle case all external torques on the whole surface can be set to zero; in contrast to that, such an external torque is inevitably applied in the antisymmetric case due to the forces which fix the separation between the particles. Total torque balance determines the asymptotic behavior of the membrane for both configurations. Particularly in the antisymmetric case, the torque is either compensated by a vertical force component (if the particles are finite) or by an offset between the two asymptotic planes (if the particles are infinitely long cylinders). Complications stemming from additional external torques (e.g., due to a magnetic field if the two particles are dipoles) have not been considered here explicitly but can be included with ease into the relevant expressions.

To see how the rather abstract expressions from the first part can be applied, the two-cylinder case ["one-dimensional (1D) problem"] has subsequently been solved in the nonlinear regime. The expressions obtained from torque balance could be used to calculate the values of the offset (antisymmetric case) and the height at the midplane (symmetric case). Finally, the force on the cylinders could be identified within the formalism as being essentially one of the parameters that had to be determined in the calculation of the profile.

The simple 1D problem already displays remarkably subtle behavior. For large deformations the small gradient approximation fails which is due to the intrinsic nonlinearity of the problem. The sign of the force, however, is predicted correctly by the approximate solution. For two spheres on the membrane (2D problem), this need not be the case: although linear calculations predict a repulsion for the symmetric configuration [20], the nonlinear expression for the force $F_{\text {sym }}$ does not rule out that the interaction between the particles is attractive for higher deformations [cf. expression for $F_{\text {sym }}$ below Eq. (23)]. While it is difficult to determine the sign analytically, coarsed-grained computer simulations suggest that a two-body attraction emerges if the deformations are high enough [46].

\section{ACKNOWLEDGMENTS}

The authors would like to thank IPAM at UCLA where part of this work was discussed. M.M.M. is also grateful for the hospitality during his stay in Mexico City. Special thanks go to Ben Reynwar and Stefan Bohlius for helpful discussions. M.D. acknowledges financial support from the German Science Foundation through an Emmy Noether grant De775/1-3. Partial support to J.G. from CONACyT Grant No. 51111 as well as DGAPA PAPIIT Grant No. IN119206-3 is acknowledged.

\section{APPENDIX A: FORCES AND TORQUES ON THE OUTER BOUNDARY}

Consider a symmetric fluid membrane with a finite number of finite-sized particles bound to it. What is the asymptotic behavior of profile properties such as the curvature $K$ and its derivatives far away from the particles?

To answer this question let us adapt a line of argument originally introduced in [23] for the case of vanishing surface tension. The excess membrane energy $E$ induced by the particles is finite. From this requirement it immediately follows that $K$ has to be square integrable and thus vanishes far away from the particles. This implies that the behavior of the membrane is determined completely by surface tension there. Thus, the membrane becomes asymptotically flat. This fact allows us to use a linearized Monge gauge to describe its behavior remote from the particles: the membrane profile is described in terms of its height $h(r, \varphi)$ above the asymptotic plane where $r$ and $\varphi$ are the cylindrical coordinates defined on that plane; we choose to place the origin in the middle of the surface region to which the particles adhere. For large $r$, the curvature $K$ is then equal to the negative (base plane) Laplacian of $h, K=-\nabla^{2} h$.

The energy can be written as $E=E_{\sigma}+E_{\kappa}+E_{\bar{\kappa}}$, where $E_{\sigma}=(\sigma / 2) \int d \varphi d r r(\nabla h)^{2}$ and $E_{\kappa}=(\kappa / 2) \int d \varphi d r r\left(\nabla^{2} h\right)^{2}$. The contribution due to the Gaussian curvature, $E_{\bar{\kappa}}$, is finite as it can be written as a topological constant plus a line integral over the geodesic curvature at the membrane boundary [32]. The energies $E_{\sigma}$ and $E_{\kappa}$ are both positive and thus finite as well. The corresponding membrane shape equation, expressed in terms of $K$, is given by $\nabla^{2} K=\lambda^{-2} K$ which has the general solution [20]

$$
\begin{aligned}
K(r, \varphi)= & a_{0} K_{0}(r / \lambda)+\sum_{n=1}^{\infty}\left(a_{n} \cos n \varphi+b_{n} \sin n \varphi\right) K_{n}(r / \lambda) \\
& +\sum_{n=1}^{\infty}\left(c_{n} \cos n \varphi+d_{n} \sin n \varphi\right) I_{n}(r / \lambda)
\end{aligned}
$$

where $I_{n}$ and $K_{n}$ are modified Bessel functions of the first and second kind, respectively [41]. The functions $I_{n}$ tend to infinity as $r \rightarrow \infty$. Thus, $c_{n}$ and $d_{n}$ must vanish. The asymptotic expansion of $K_{n}$ for large $r$ is given by

$$
K_{n}(r)=\sqrt{\frac{\pi}{2 r}} e^{-r}\left(1+\frac{4 n^{2}-1}{8 r}+\cdots\right)
$$

and shows that the functions $K_{n}(r)$ decay essentially exponentially for increasing $r$.

The force $\boldsymbol{F}_{\text {out }}$ on the outer boundary is a line integral over the appropriate projection of the stress tensor, 
$\int_{\partial \Sigma_{\text {out }}} d s l_{d} f^{a}$. Since $l_{a} f^{a}$ depends on derivatives and powers of $K$ plus a surface tension term [14], the contribution to the force due to curvature vanishes as $r \rightarrow \infty$. The only surviving contribution at infinity is a horizontal pulling force proportional to $\sigma$.

Although the vertical force vanishes at infinity, an external torque $\boldsymbol{M}_{\text {out }}$ may still act on the outer membrane boundary. The reason for this is that the length of the lever arm increases linearly with $R$. The origin of the torque is the surface tension, and it is given by

$$
\begin{aligned}
\boldsymbol{M}_{\mathrm{out}}= & \sigma \int_{0}^{2 \pi} d \varphi R[R \boldsymbol{r}+h(R, \varphi) z] \times \boldsymbol{l} \\
= & \sigma \int_{0}^{2 \pi} d \varphi R\left\{-h(R, \varphi) l_{\varphi} \boldsymbol{r}\right. \\
& +\left[h(R, \varphi) l_{r}-R l_{z}\right] \varphi+R l_{\varphi} z,
\end{aligned}
$$

where $\boldsymbol{l}=l_{r} \boldsymbol{r}+l_{\varphi} \boldsymbol{\varphi}+l_{z} z$ in cylindrical coordinates with basis vectors $\{\boldsymbol{r}, \boldsymbol{\varphi}, \boldsymbol{z}\}$. The unit vectors $\boldsymbol{r}$ and $\boldsymbol{\varphi}$ lie on the reference plane whereas $z$ is normal to it. The moving trihedron of the boundary curve consists of the vectors $\boldsymbol{l}, \boldsymbol{t}$, and $\boldsymbol{n}$ which are perpendicular to each other. In particular, $\boldsymbol{l}$ may be expanded in terms of the height function $h$ and its derivatives:

$$
\boldsymbol{l}=\frac{\boldsymbol{r}-\left.\frac{1}{q R}\left[\left(\partial_{\varphi} h\right)\left(\partial_{r} h\right)\right]\right|_{r=R} \boldsymbol{\varphi}+\left.\frac{1}{q}\left(\partial_{r} h\right)\right|_{r=R} \boldsymbol{z}}{\sqrt{1+\left\{\left.\frac{1}{q R}\left[\left(\partial_{\varphi} h\right)\left(\partial_{r} h\right)\right]\right|_{r=R}\right\}^{2}+\left\{\left.\frac{1}{q}\left(\partial_{r} h\right)\right|_{r=R}\right\}^{2}}},
$$

where $q:=1+\left[\frac{\left.\left(\partial_{\varphi} h\right)\right|_{r=R}}{R}\right]^{2}$.

The height function $h(r, \varphi)$ satisfies the linearized shape equation $\nabla^{2}\left(\nabla^{2} h\right)=\lambda^{-2} \nabla^{2} h$. The solution of this equation consistent with a finite value for $E_{\sigma}$ is [20]

$$
\begin{aligned}
h(r, \varphi)= & C_{0}+A_{0} K_{0}(r / \lambda) \\
& +\sum_{n=1}^{\infty}\left(A_{n} \cos n \varphi+B_{n} \sin n \varphi\right) K_{n}(r / \lambda) \\
& +\sum_{n=1}^{\infty}\left(C_{n} \cos n \varphi+D_{n} \sin n \varphi\right) r^{-n} .
\end{aligned}
$$

Inserting Eqs. (A4) and (A5) into the equation of the torque, Eq. (A3), one obtains

$$
\boldsymbol{M}_{\mathrm{out}}=\sigma \int_{0}^{2 \pi} d \varphi R\left[\frac{2\left(C_{1} \cos \varphi+D_{1} \sin \varphi\right)}{R} \boldsymbol{\varphi}+O\left(\frac{1}{R^{2}}\right)\right] .
$$

The unit vector $\varphi$ still depends on the coordinate $\varphi$. To evaluate the integral over $\varphi$ in Eq. (A6), we rewrite $\varphi$ as $\boldsymbol{\varphi}=-\sin \varphi \boldsymbol{x}+\cos \varphi \boldsymbol{y}$, where $\boldsymbol{x}$ and $\boldsymbol{y}$ are the Cartesian coordinates parallel to the asymptotic plane. We finally obtain for $R \rightarrow \infty$

$$
\boldsymbol{M}_{\text {out }}=2 \pi \sigma\left(-D_{1} \boldsymbol{x}+C_{1} \boldsymbol{y}\right) .
$$

Hence, the horizontal torque is in general not equal to zero even though the vertical force is. Its contributions stem from those terms of the height function (A5) which are proportional to $r^{-1}$ and decay slowest for increasing distance from the particles. Conversly, the existence of an external torque forces the surface to display a very slow $1 / r$ decay.

\section{APPENDIX B: CALCULATIONS ON THE LINEARIZED LEVEL}

In this appendix we will show that Eq. (28) is consistent with the results of Ref. [21]. To this end we calculate $h(0)=-h_{0}$ and compare it with the right-hand side of Eq. (28).

Due to the symmetry, it is sufficient to consider only the left cylinder. Its axis is located at $x=-d / 2$ (see Fig. 3). For $-d / 2-\delta_{0} \leq x \leq-d / 2+\delta_{\mathrm{i}}$, cylinder and membrane are in contact $\left(\delta_{\mathrm{i}, \mathrm{o}}=R \sin \alpha_{\mathrm{i}, 0}\right)$. In small gradient approximation the shape of the outer membrane segment $\left(x \leq d / 2-\delta_{0}\right)$ is given by $[21]$

$$
h_{\text {out }}(x)=B \exp (-|x+d / 2| / \lambda),
$$

where $B=-\delta_{0} \lambda / R$ to first order in $\delta_{0}$ (strictly speaking, the expansion is in $\delta_{\mathrm{o}} / \lambda$ here and below). The membrane segment adhering to the left cylinder has the circular profile

$$
h_{\text {cyl }}(x)=-\left[h_{\mathrm{c}}+\sqrt{R^{2}-(x+d / 2)^{2}}\right],
$$

where $h_{\mathrm{c}}$ is defined in Fig. 3. The profile has to be continuous at $x=-d / 2-\delta_{0}$. This condition yields

$$
\begin{aligned}
h_{\mathrm{c}} & =-\sqrt{R^{2}-\delta_{\mathrm{o}}^{2}}+\frac{\delta_{\mathrm{o}} \lambda}{R} \exp \left(-\delta_{\mathrm{o}} / \lambda\right)=-R+\frac{\lambda}{R} \delta_{\mathrm{o}}+O\left(\delta_{\mathrm{o}}^{2}\right) \\
& =-R+\frac{\lambda \operatorname{coth} \frac{d}{2 \lambda}}{R} \delta_{\mathrm{i}}+O\left(\delta_{\mathrm{i}}^{2}\right) .
\end{aligned}
$$

In the last step we exploited the fact that $\delta_{0}=\operatorname{coth}\left(\frac{d}{2 \lambda}\right) \delta_{\mathrm{i}}$ to first order [21].

The profile of the membrane between the two cylinders in the symmetric case is given by [21]

$$
h_{\text {in }}(x)=C+D \cosh (x / \lambda),
$$

where $C$ and $D$ are constants that can be determined from the conditions of continuous profile and slope at $x=-d / 2+\delta_{\mathrm{i}}$. The latter condition yields

$$
D=\frac{\lambda \delta_{\mathrm{i}}}{\sqrt{R^{2}-\delta_{\mathrm{i}}^{2}}} \cdot \frac{1}{\sinh \left(\frac{-\frac{d}{2}+\delta_{i}}{\lambda}\right)}=-\frac{\lambda \delta_{\mathrm{i}}}{R \sinh \left(\frac{d}{2 \lambda}\right)}+O\left(\delta_{\mathrm{i}}^{2}\right),
$$


the former

$$
\begin{aligned}
& C=-\left(h_{\mathrm{c}}+\sqrt{R^{2}-\delta_{i}^{2}}\right)-D \cosh \left(\frac{-\frac{d}{2}+\delta_{\mathrm{i}}}{\lambda}\right) \\
& \stackrel{\lambda \operatorname{coth}\left(\frac{d}{2 \lambda}\right)}{R} \delta_{\mathrm{i}}+O\left(\delta_{\mathrm{i}}^{2}\right)=O\left(\delta_{\mathrm{i}}^{2}\right) .
\end{aligned}
$$

To first order the depth $h_{0}$ at the midline of the profile [47] is thus given by

$$
h_{0}=-h_{\mathrm{in}}(0)=-(C+D)=\frac{\lambda \delta_{\mathrm{i}}}{R \sinh \left(\frac{d}{2 \lambda}\right)}+O\left(\delta_{\mathrm{i}}^{2}\right)
$$

It can also be obtained from Eq. (28). Using $K_{\perp}=-h_{\text {in }}^{\prime \prime}(0)$ $=-\frac{D}{\lambda^{2}}$ yields

$$
h_{0}=-\frac{D+\frac{D^{2}}{2 \lambda^{2}} h_{\mathrm{c}}}{1-\frac{D^{2}}{2 \lambda^{2}}}=\frac{\lambda \delta_{\mathrm{i}}}{R \sinh \left(\frac{d}{2 \lambda}\right)}+O\left(\delta_{\mathrm{i}}^{2}\right) .
$$

The two results coincide at first order which confirms the validity of Eq. (28) within this approximation.
[1] H. Lodish, A. Berk, S. L. Zipursky, P. Matsudaira, D. Baltimore, and J. Darnell, Molecular Cell Biology (Freeman, New York, 2000).

[2] Handbook of Biological Physics, edited by R. Lipowsky and E. Sackmann (Elsevier, Amsterdam, 1995), Vol. 1.

[3] Endocytosis, edited by M. Marsh, Frontiers in Molecular Biology (Oxford University Press, Oxford, 2001).

[4] M. S. Robinson, Trends Cell Biol. 7, 99 (1997).

[5] H. T. McMahon and J. L. Gallop, Nature (London) 438, 590 (2005).

[6] B. Antonny, Curr. Opin. Struct. Biol. 18, 386 (2006).

[7] E. J. Luna and A. L. Hitt, Science 258, 955 (1992).

[8] U. Seifert, Adv. Phys. 46, 13 (1997).

[9] R. Capovilla and J. Guven, J. Phys. A 35, 6233 (2002).

[10] J. Guven, J. Phys. A 37, L313 (2004).

[11] M. A. Lomholt and L. Miao, J. Phys. A 39, 10323 (2006).

[12] M. M. Kozlov, J. Phys.: Condens. Matter 18, S1177 (2006).

[13] M. M. Müller, M. Deserno, and J. Guven, Europhys. Lett. 69, 482 (2005).

[14] M. M. Müller, M. Deserno, and J. Guven, Phys. Rev. E 72, 061407 (2005).

[15] A. Dominguez, M. Oettel, and S. Dietrich, e-print arXiv:condmat/0611329.

[16] P. A. Kralchevsky, V. N. Paunov, N. D. Denkov, and K. Nagayama, J. Chem. Soc., Faraday Trans. 91, 3415 (1995); P. A. Kralchewsky and K. Nagayama, Adv. Colloid Interface Sci. 85, 145 (2000).

[17] I. Koltover, J. O. Rädler, and C. R. Safinya, Phys. Rev. Lett. 82, 1991 (1999).

[18] M. Goulian, R. Bruinsma, and P. Pincus, Europhys. Lett. 22, 145 (1993); 23, 155(E) (1993); note also the further correction in J.-B. Fournier and P. G. Dommersnes, ibid. 39, 681 (1997).

[19] V. I. Marchenko and C. Misbah, Eur. Phys. J. E 8, 477 (2002); D. Bartolo and J.-B. Fournier, ibid. 11, 141 (2003).

[20] T. R. Weikl, M. M. Kozlov, and W. Helfrich, Phys. Rev. E 57, 6988 (1998).

[21] T. R. Weikl, Eur. Phys. J. E 12, 265 (2003).

[22] P. G. Dommersnes, J.-B. Fournier, and P. Galatola, Europhys. Lett. 42, 233 (1998).
[23] K. S. Kim, J. Neu, and G. Oster, Biophys. J. 75, 2274 (1998).

[24] P. Biscari, F. Bisi, and R. Rosso, J. Math. Biol. 45, 37 (2002); P. Biscari and F. Bisi, Eur. Phys. J. E 7, 381 (2002).

[25] P.-G. de Gennes, F. Brochard-Wyart, and D. Quere, Capillarity and Wetting Phenomena (Springer, Berlin, 2003).

[26] P. B. Canham, J. Theor. Biol. 26, 61 (1970).

[27] W. Helfrich, Z. Naturforsch. C 28693 (1973).

[28] M. Nizette and A. Goriely, J. Math. Phys. 40, 2830 (1999).

[29] M. Doi and S. F. Edwards, The Theory of Polymer Dynamics (Cambridge University Press, Cambridge, England, 1986), Sec. 8.8.

[30] O. Kratky and G. Porod, Rec. Trav. Chim. 68, 1106 (1949).

[31] I. M. Kulic, Ph.D. thesis, University of Mainz, 2004 (unpublished).

[32] For a detailed introduction to the differential geometry of twodimensional surfaces see M. Do Carmo, Differential Geometry of Curves and Surfaces (Prentice-Hall, Englewood Cliffs, NJ, 1976); E. Kreyszig, Differential Geometry (Dover, New York, 1991).

[33] T. Frankel, The Geometry of Physics (Cambridge University Press, Cambridge, England, 2003).

[34] J.-B. Fournier, Soft Mater. 3, 883 (2007); see also e-print arXiv:cond-mat/0702279.

[35] D. Stamou, C. Duschl, and D. Johannsmann, Phys. Rev. E 62, 5263 (2000); J.-B. Fournier and P. Galatola, ibid. 65, 031601 (2002).

[36] G. Arreaga, R. Capovilla, C. Chryssomalakos, and J. Guven, Phys. Rev. E 65, 031801 (2002).

[37] L. D. Landau and E. M. Lifshitz, Mechanics, 3rd ed. (Butterworth-Heinemann, Oxford, 1976).

[38] The shape of the interface between two incompressible fluids under gravity (with density difference $\Delta \rho>0$ and gravitational acceleration $g$ ) is determined by the Young-Laplace law which states that the hydrostatic pressure $P=-\Delta \rho g h$ equals the interfacial tension $\sigma$ times the curvature $K: \dot{\psi}=h / \ell^{2}$, with $\ell$ $=\sqrt{\sigma /(g \Delta \rho)}$. By differentiating this equation and equating it with $\dot{h}=\sin \psi$ we obtain Eq. (35) where $\lambda=\ell$ and $\eta=1$.

[39] The other solution with $\alpha_{\mathrm{o}}<0$ and $\dot{\psi} \geq 0$ can be simply obtained by a reflection on the $(x, y)$ plane. 
[40] The expression for $\eta<1$ in Eq. (40) is valid for $0 \leq \tilde{s}$ $\leq \frac{2}{\sqrt{\eta}} K\left(\frac{1}{m}\right)$.

[41] Handbook of Mathematical Functions, 9th ed., edited by M. Abramowitz and I. A. Stegun (Dover, New York, 1970).

[42] L. D. Landau and E. M. Lifshitz, Theory of Elasticity, 3rd ed. (Butterworths-Heinemann, Oxford, 1986).

[43] U. Seifert and R. Lipowsky, Phys. Rev. A 42, 4768 (1990).
[44] M. Deserno, M. M. Müller, and J. Guven, Phys. Rev. E 76, 011605 (2007).

[45] The intermediate maximum is not considered in the following as it does not correspond to a stable profile.

[46] B. J. Reynwar et al., Nature (London) 447, 461 (2007).

[47] Note that $h_{0}$ is defined differently in Ref. [21]; there it is given by $-\left(h_{\mathrm{c}}+R\right)$. 